\title{
Petrogenesis, U-Pb and Sm-Nd geochronology of the Furna Azul Migmatite: partial melting evidence during the San Ignácio Orogeny, Paraguá Terrane, SW Amazon Craton
}

\author{
Petrogênese, geocronologia U-Pb e Sm-Nd do Migmatito Furna Azul: \\ evidência de fusão parcial durante a Orogenia San Ignácio, Terreno \\ Paraguá, SW do Cráton Amazônico
}

\author{
Newton Diego Couto do Nascimento ${ }^{1,6,7 *}$, Amarildo Salina Ruizz ${ }^{2,6,7}$, \\ Ronaldo Pierosan ${ }^{3,7}$, Gabrielle Aparecida de Lima ${ }^{4,6,7}$, João Batista Matos, ${ }^{3,6,7}$, \\ Jean-Michel Lafon ${ }^{5,7}$, Candido Augusto Veloso Moura ${ }^{5,7}$
}

\begin{abstract}
The Furna Azul Migmatite is a $\sim 10 \mathrm{~km}^{2}$ complex located in Pontes e Lacerda city, Mato Grosso, Brazil. It belongs to Paraguá Terrane, limit with Rio Alegre Terrane, southeast of San Ignacio Province, in Amazon Craton. It consists of transitional metatexites with amphibolite enclaves and dioritic injections. The rocks were divided in residuum rich and leucosome rich; both have three deformation phases marked by folded stromatic layers affected by spaced foliation and metamorphosed in amphibolite facies, represented by garnet, biotite, sillimanite, and by the clinopyroxene in the enclaves. The metamorphic retrograde to greenschist is marked by formation of chlorite, muscovite and prehnite. Residuum-rich metatexites show higher $\mathrm{CaO}$ and $\mathrm{Na}_{2} \mathrm{O}$ contents, separating them from $\mathrm{K}_{2} \mathrm{O}, \mathrm{Ba}$ and $\mathrm{Rb}$ enriched transitional metatexites. $\mathrm{U}-\mathrm{Pb}$ on zircon and $\mathrm{Sm}-\mathrm{Nd}$ whole-rocks dating indicates that the residuum-rich metatexite crystallized at $1436 \pm 11 \mathrm{Ma}$, with a $\mathrm{T}_{\mathrm{DM}}$ age of $1.90 \mathrm{Ga}$ and $\varepsilon_{\mathrm{Nd}(1.43)}$ of -0.54 , whereas the dioritic injection crystallized at $1341,7 \pm 17$ Ma with a $\mathrm{T}_{\mathrm{DM}}$ age of $1.47 \mathrm{Ga}$ and $\varepsilon_{\mathrm{Nd}(1.34)}$ of 3.39. These results indicate that the Furna Azul Migmatite protolith was formed during the San Ignácio Orogeny and was reworked during the same orogeny, as basement for collisional to post-magmatic granites from Pensamiento Intrusive Suite.
\end{abstract}

KEYWORDS: Furna Azul Migmatite; Paraguá Terrane; Rondonian-San Ignacio Province.

\begin{abstract}
RESUMO: O Migmatito Furna Azul é um complexo de $-10 \mathrm{~km}^{2}$ localizado em Pontes e Lacerda, Mato Grosso, Brasil. Pertence do Terreno Paraguá, próximo ao limite com o Terreno Rio Alegre, sudeste da Província Rondoniana - San Ignácio - Cráton Amazônico. O migmatito consiste de metatexitos transicionais com enclaves anfibolíticos e injeçōes dioríticas. Os metatexitos são distinguidos em ricos em resíduo e ricos em leucossoma e exibem três fases deformacionais marcadas pelo bandamento estromático dobrado afetado por uma xistosidade espaçada e metamorfisados na fácies anfibolito alto, representada por granada, biotita e sillimanita, bem como pela formação de clinopiroxênio nos enclaves. O retrometamorfismo para a fácies xisto verde é marcado pela formação de clorita, muscovitalsericita e prehnita. O metatexito rico em resíduo apresenta maiores teores de $\mathrm{CaO}, \mathrm{Na}_{2} \mathrm{O}$, separando-os do metatexito transicional enriquecidos em $\mathrm{K}_{2} \mathrm{O}, \mathrm{Ba}$ e $\mathrm{Rb}$. Comparando com produtos de anatexia, nota-se uma afinidade com produtos de protólitos tonalíticos elou anfibolíticos. Os dados geocronológicos (U-Pb SHRIMP em zircão e Sm-Nd em rocha total) mostraram que o metatexito rico em resíduo teve sua cristalizaçāo em $1436 \pm$ $11 \mathrm{Ma}$, com idade modelo $T_{D M}$ de 1,90 Ga e $\varepsilon_{N d(43)}$ de -0,54, enquanto a injeção diorítica cristalizon em $1341.7 \pm 17 \mathrm{Ma}$ com idade modelo $T_{D M}$ de 1,47 Ga e $\varepsilon_{\text {Nd(1.34) }}$ de 3,39. Esses resultados evidenciam que o protólito do Migmatito Furna Azul teria sido formado durante a Orogenia San Ignácio $(1.43 \mathrm{Ga})$ posteriormente retrabalhado, servindo de embasamento para o magmatismo tardi a pós-colisional representado pela Suite Intrusiva Pensamiento.
\end{abstract}

PALAVRAS-CHAVE: Migmatito Furna Azul; Terreno Paraguá; Provincia Rondoniana - San Ignácio.

\footnotetext{
${ }^{1}$ Geoscience Postgraduate Program, Institute of Exact and Earth Sciences, Universidade Federal do Mato Grosso - UFMT, Cuiabá (MT), Brazil. E-mail: newtongeologia@hotmail.com

2Department of General Geology, Institute of Exact and Earth Sciences, Universidade Federal do Mato Grosso - UFMT, Cuiabá (MT), Brazil. E-mail: asruiz@gmail.com ${ }^{3}$ Department of Mineral Resources, Universidade Federal do Mato Grosso - UFMT, Cuiabá (MT), Brazil. E-mail: ronaldo.pierosan@yahoo.com.br ${ }^{4}$ Institute of Engineering, Universidade Federal do Mato Grosso - UFMT, Várzea Grande (MT), Brazil. E-mail: gabilimagel@gmail.com 5Isotope Geology Laboratory, Institute of Geosciences, Universidade Federal do Pará - UFPA, Belém (PA), Brazil. E-mail: lafonjm@ufpa.br ${ }^{6}$ Research Group Crustal Evolution and Tectonics: Guaporé, Universidade Federal do Mato Grosso - UFMT, Cuiabá (MT), Brazil. E-mail: prof.jmatos@gmail.com ${ }^{7}$ National Institute of Science and Geoscience Technology of the Amazon - GEOCIAM, Universidade Federal do Pará - UFPA, Belém (PA), Brazil. E-mail: candido@ufpa.br *Corresponding author.

Manuscrip ID: 20160030. Received in: 02/25/2016. Approved in: 05/09/2016.
} 


\section{INTRODUCTION}

Several geotectonic reconstructions of the RondonianSan Ignacio Province in southwestern Amazon Craton take into account the amalgamation of intra-oceanic volcanic arcs and the development of continental arcs, attributed to the closure of oceans and microcontinent-continent collisions, including late to post-collisional stages of orogenic collapse in a tectonically active Mesoproterozoic margin (Cordani et al. 1979, 2009, Teixeira et al. 1989, 2010, Tassinari \& Macambira 1999, Geraldes et al. 2001, Ruiz 2005, Bettencourt et al. 2010). In this context of successive continental agglutinations, the Paraguá Terrane, an allochthonous microcontinent, collided with the Amazon protoCraton during the San Ignacio Orogeny $(1.56-1.30 \mathrm{Ga})$ (Bettencourt et al. 2010, Ruiz et al. 2011).

The Paraguá Terrane is composed of granulites, paragneisses and orthogneisses, migmatitic gneisses and schists, whose ages between 1800 and 1600 Ma were yielded by geochronological data (Litherland et al. 1986, Boger et al. 2005, Santos et al. 2008, Figueiredo et al. 2013, Faria et al. 2014).

The stratigraphic sequence proposed by Litherland $e t a l$. (1986) consists of the Lomas Manechis Granulite Complex (ca. $1900 \mathrm{Ma}$ ), followed by an association of migmatitic gneisses of the Chiquitania Gneiss Complex. The younger San Ignacio Schists Group established a stratigraphic sequence in which the Chiquitania Gneiss Complex represents a sedimentary, orogenic event that started after 1690 Ma (Boger et al. 2005). The Lomas Manechis Granulite Complex consists of a granite-gneiss suite bearing amphibole and orthopyroxene originated between 1690 and 1660 Ma. Proposed models consider the sequences to be granulitized and migmatized during the San Ignacio Orogeny, and magmatism to have taken place in a magmatic arc setting represented by several orogenic granitoids emplaced around 1300 Ma (Matos et al. 2008, 2009, Jesus et al. 2010, Ruiz et al. 2012, Nalon et al. 2013, França et al. 2014).

The Furna Azul Migmatite (FAM), as defined by Nascimento et al. (2013), was first correlated to the gneisses of Serra do Baú Complex (Ruiz 2005). This unit is composed by othogneisses stratigraphically correlated to the gneisses of Chiquitania Gneiss Complex, which outcrop mainly close to Santa Barbara cliffs and Ricardo Franco ridges, in Mato Grosso state.

The purpose of this paper was to discuss the petrogenesis of the FAM and to temporally establish the metamorphic episodes that allowed the melting event, based on geochronological data.

Concepts of Mehnert (1968) on migmatite, which evolved from Sederholm (1907) and Holmquist (1916), are first for the migmatite issue. Sawyer (2008) suggested that protholiths that undergo partial melting provide neosome as their main product. Neosome is comprised of a solid residual portion (residuum) and a silicate melt, which is the precursor magma (leucosome). The author proposed that, in a migmatitic system, the portion that does not undergo partial melting and consists essentially of refractory minerals is called paleosome. In this paper, the concepts and systematic classification by Sawyer (2008) have been applied.

\section{REGIONAL GEOLOGICAL CONTEXT}

The Amazon Craton is one of the largest cratonic areas in the world. It is located in the north-northwestern region of South America and comprises the largest Precambrian segment of the South American Platform (Fuck et al. 2008, Cordani et al. 2009, Brito Neves 2011). It is divided in Central Brazil and Guyana shields by the Amazon Basin and it is surrounded by Neoproterozoic-Cambrian and Andean mobile belts (Tassinari \& Macambira 1999).

The concept of geochronological province proposed by Cordani et al. (1979) describes Proterozoic mobile belts around the Archean stable cores. This model has been recurrently updated and the subdivision has been refined (Teixeira et al. 1989, Tassinari \& Macambira 1999, Tassinari et al. 2000, Cordani \& Teixeira 2007) in the following provinces of the Amazon Craton (Fig. 1A): Central Amazonian ( $2.60 \mathrm{Ga}$ ); Maroni-Itacaiunas (2.25 to $2.05 \mathrm{Ga}$ ); VentuariTapajós (1.98 to $1.81 \mathrm{Ga}$ ); Rio Negro-Juruena (1.78 to $1.56 \mathrm{Ga})$; Rondonian-San Ignácio (1.56 to $1.30 \mathrm{Ga})$; and Sunsás-Aguapeí (1.28 to $0.95 \mathrm{Ga}$ ).

The study area is located in the south-southeastern portion of the Rondonian-San Ignácio Province, considered by Bettencourt et al. (2010) as an orogen composed of Paleo and Mesoproterozoic terranes. These terranes are defined as the latest stage of cratonization during the Meso-Neoproterozoic, mainly in the regions where the Sunsás-Aguapeí Orogeny rework was less intense. They enabled the recognition of features of the San Ignácio Orogeny and identified four terranes (Fig. 1B): Jauru (1.78 - 1.42 Ga), Rio Alegre (1.51 $1.38 \mathrm{Ga})$, Alto Guaporé $(<1.51-1.33 \mathrm{Ga})$ and Paraguá $(1.74-1.32 \mathrm{Ga})$.

Discussions on the stratigraphic and deformational outline of the Paraguá Terrane have been carried out since the first studies (Litherland \& Bloomfield 1981, Litherland et al. 1986), into recent studies (Boger et al. 2005, Santos et al. 2008, Matos Salinas 2010, Bettencourt et al. 2010). In the area, the basement of the Aguapeí Group is composed by migmatitic gneisses, with some occurrences of granulites with ages ranging from 1711 to $1640 \mathrm{Ma}$ (Figueiredo et al. 2013, Matos et al. 2013, Faria et al. 2014). These rocks crop out as 


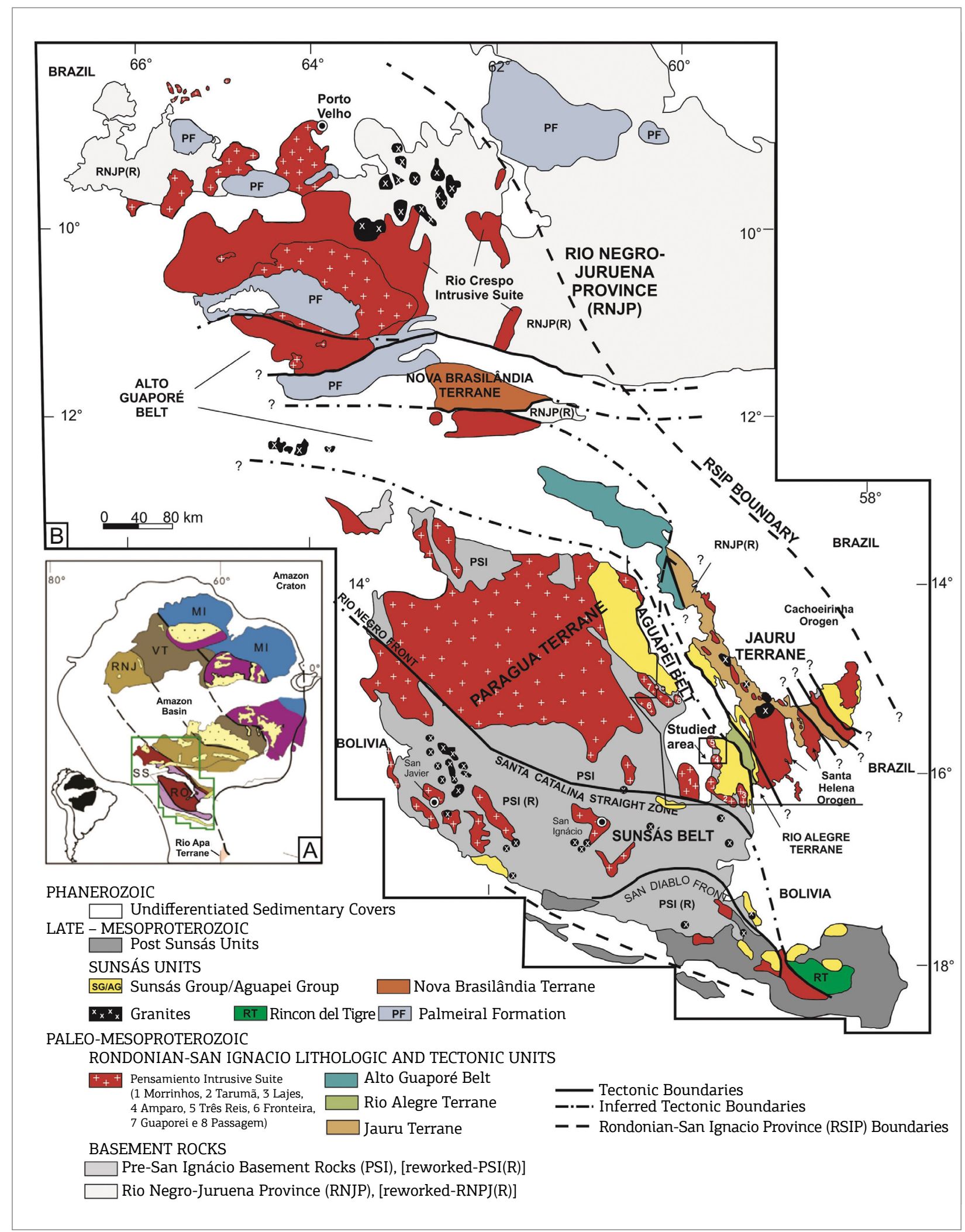

Figure 1. (A) Geochronological provinces of the Amazon Craton (Cordani \& Teixeira 2007): MI - MaroniItacaiunas; VT - Ventuari-Tapajós; RNJ - Rio Negro-Juruena; RO - Rondonian-San Ignácio; SS - Sunsás-Aguapeí; (B) geological sketchmap of SW Amazon Craton showing the approximate boundaries of the main provinces, orogens, terranes, mobile belts, tectonic elements, and geological units. Modified from Bettencourt et al. (2010) and França et al. (2014). 
mega-xenoliths or as basement windows in the midst of several granitic intrusions from San Ignácio Orogeny (Geraldes et al. 2001, Matos et al. 2009, Jesus et al. 2010, Ruiz et al. 2012, Nalon et al. 2013, França et al. 2014) (Fig. 2A and Tab. 1). The orthogneisses of this region were grouped in the Serra do Baú Complex by Ruiz (2005) and are considered as high-K calc-alkaline protholiths of metaluminous to peraluminous character emplaced in a magmatic arc setting due to subduction of a Paleoproterozoic oceanic crust, and later reworked during the San Ignácio Orogeny (Figueiredo et al. 2013, Faria et al. 2014).

\section{FIELD AND PETROGRAPHIC ASPECTS}

The FAM is partially covered by the metasedimentary rocks of the Aguapeí Group and by sediments of the Guaporé Formation. It is in abrupt contact with the Amparo Granite (Fig. 2B). Granitoid dikes, amphibolitic enclaves and dioritic injections are commonly observed.
Migmatites consist of rocks with heterogeneous composition and texture that show evidence of ductile and ductile-ruptile deformation by three deformation phases. The first phase consists of a millimeter-size compositional banding $\left(S_{1}\right)$ in residuum-rich rocks. Granitic leucosome occurs parallel to the compositional banding or cross-cutting it. Both structures are deformed, by nearly isoclinal folds that were developed during the second deformation phase. This deformation phase is associated with transposition and development of an schistosity $\left(\mathrm{S}_{2}\right)$ towards northwest, ranging between 85 and $90^{\circ}$, dipping to northeast and southwest (Fig. 3A). The third deformation phase is marked by a gentle folding, spaced foliation $\left(S_{3}\right)$ of kind crenulation cleavage and shear zone with oblique-transcurrent component in the northeast direction and with southeast dips (Fig. 3B).

Based on field works and petrographic characterization, leucosome-rich transitional metatexites (Fig. 3B) and residuum-rich metatexites (Fig. 3C) were identified using the Sawyer (2008) classification scheme.

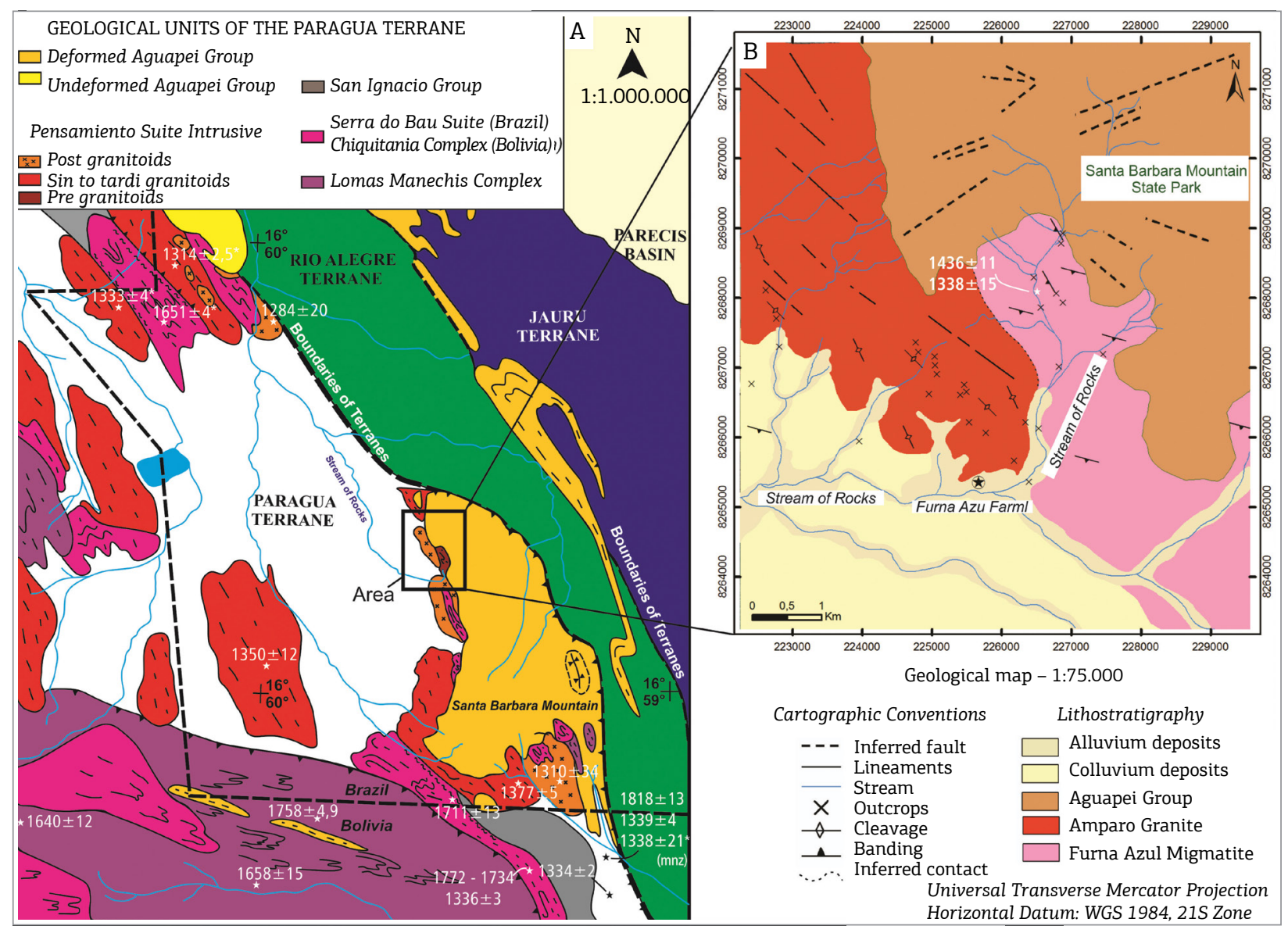

Figure 2. (A) Geological map of the Paraguá Terrane modified from Ruiz et al. (2011). The dashed line represents the boundary between Brazil and Bolivia - southwestern Mato Grosso; (zr) and (mnz) are zircon and monazite, respectively; (B) detailed geological map of the study area. 


\section{Residuum-rich metatexite}

The residuum-rich metatexite is gray, leucocratic to mesocratic, with centimeter veins of granitic leucosome. It is parallel to the first deformation phase foliation and crosscut by late leucosome veins, which, in turn, are controlled by the main crenulation axis generated during the second deformation phase. It is fine grained with few grains that reach up to $2.0 \mathrm{~mm}$, and the texture is granolepidoblastic composed by quartz (32\%), plagioclase (29\%), biotite (28\%) and garnet (9\%). Zircon, orthoclase, apatite, titanite and opaque are the accessory minerals while chlorite, muscovite/sericite, prehnite, clay minerals and epidote compose the retrometamorphic assemblage. The residuum-rich metatexite presents preserved garnet grains, while in other lithotypes its pseudomorphosed, suggesting higher interaction with the leucosome.

Quartz forms xenoblastic grains of different sizes, with irregular borders and strong wavy extinction. Garnet is the main inclusion phase. Plagioclase (andesine) is xenoblastic with irregular borders and orthogonal or oblique twining to the $\mathrm{C}$ axis, suggesting boundary consumption. Biotite displays a subidioblastic aspect and evidence of reaction to prehnite, muscovite and chlorite. Garnet is granular and disseminated poikiloblastic, envelops anhedral grains of quartz, and displays strain shadows composed of biotite (Fig. 4A), as well as chlorite parallel to the schistosity.

\section{Transitional leucosome-rich metatexite}

This lithotype is composed of leucocratic rocks generated by the interaction between leucosomatic and mafic-rich residuum levels. The grain size is medium and it displays an aligned arrangement of quartz (35\%), orthoclase (28\%) and plagioclase $(16 \%)$, with interstitial biotite $(12 \%)$ and disseminated peritectic garnet $(4 \%)$. The accessory assemblage consists of epidote, monazite, zircon, apatite, and opaque minerals, while muscovite/sericite, carbonate, chlorite, prehnite and rutile represent the secondary assemblage.

Quartz is stretched with irregular borders and deformation lamella with wavy extinction. Quartz sometimes occurs as interstitial drops resulting from a melting reaction (Fig. 4B). Orthoclase usually occurs in anhedral to subhedral shapes and rarely displays twinning. Moreover, orthoclase has plagioclase drops and perthite intergrowth. Plagioclases are $<1.0 \mathrm{~mm}$, with irregular borders that crosscut albite and pericline twinning terminations. This suggests consumption by perithetic phase reaction. In other situations, plagioclase occurs associated to biotite as a product of garnet reaction, suggesting pseudomorphism (Fic. 4C), or

Table 1. Geochronological and isotopic data for the basement rocks of Paraguá Terrane in the southwestern region of Mato Grosso.

\begin{tabular}{|c|c|c|c|c|c|c|}
\hline \multirow{2}{*}{$\begin{array}{l}\text { MÉTHOD } \\
\text { TOPONYMY }\end{array}$} & \multicolumn{2}{|c|}{ U-Pb (Ma) } & \multirow{2}{*}{$\begin{array}{c}\mathrm{Pb}-\mathrm{Pb}(\mathrm{Ma}) \\
\text { Magmatism }\end{array}$} & \multicolumn{2}{|c|}{ Sm-Nd } & \multirow{2}{*}{ REFERENCES } \\
\hline & Magmatism & Metamorphism & & $\mathrm{T}_{(\mathrm{DM})}(\mathrm{Ga})$ & $\varepsilon_{\mathrm{Nd}(\mathrm{t})}$ & \\
\hline Passagem Granite & $1284 \pm 20$ & & & 1,60 & -7 & Jesus et al. (2010) \\
\hline Guaporeí Granite & & & $1314 \pm 2,5$ & 1,76 & -14 & Nalon et al. (2013) \\
\hline Lajes Granite & $1310 \pm 34$ & & & 1,69 & & Geraldes et al. (2001) \\
\hline Fronteira Granite & & & $1333 \pm 4$ & & & Ruiz et al. (2012) \\
\hline Morrinhos Granite & $1350 \pm 12$ & & & 1,77 & $-2,57$ & França et al. (2014) \\
\hline Tarumã Granite & $1375 \pm 5$ & & & 1,90 & $-4,11$ & Matos et al. (prep..) \\
\hline \multirow{3}{*}{$\begin{array}{l}\text { Lomas Manechi } \\
\text { Granulite }\end{array}$} & $1640 \pm 12$ & & & & & \multirow{3}{*}{ Matos et al. (2013) } \\
\hline & $1658 \pm 15$ & & & & & \\
\hline & $1758 \pm 4,9$ & & & & & \\
\hline Turvo Gneiss & & & $1651 \pm 4$ & & & $\begin{array}{l}\text { Figueiredo et al. } \\
\text { (2013) }\end{array}$ \\
\hline Rio Fortuna Gneiss & $1711 \pm 13$ & & & & & Faria et al. (2014) \\
\hline Rio Fortuna Gneiss & $1772-1734$ & $1336 \pm 3$ & & 2,06 & $-3,86$ & Santos et al. (2008) \\
\hline $\begin{array}{l}\text { Lomas Manechi } \\
\text { Granulite }\end{array}$ & $1818 \pm 13$ & $\begin{array}{c}1339 \pm 4 \\
1334 \pm 21(\mathrm{zr}) \\
1338 \pm 21(\mathrm{mnz})\end{array}$ & & 2,07 & 0,57 & Santos et al. (2008) \\
\hline
\end{tabular}


as subhedral tabular grains with evidence of sericitization, argillization and carbonation.

\section{Schlieren-bearing leucosome}

Schlieren-bearing leucosome is usually found as centimeter-sized hololeucocratic bands with biotite and garnet schlieren. It is medium- to coarse-grained and exhibits an inequigranular texture composed of orthoclase (45\%), quartz $(30 \%)$, plagioclase $(20 \%)$, and biotite + garnet $(<5 \%)$.

Orthoclase is subhedral and larger than $3.0 \mathrm{~mm}$, twinless, with string perthite and granular quartz and biotite inclusions (Fig. 4D). Quartz is interstitial in feldspar grains or
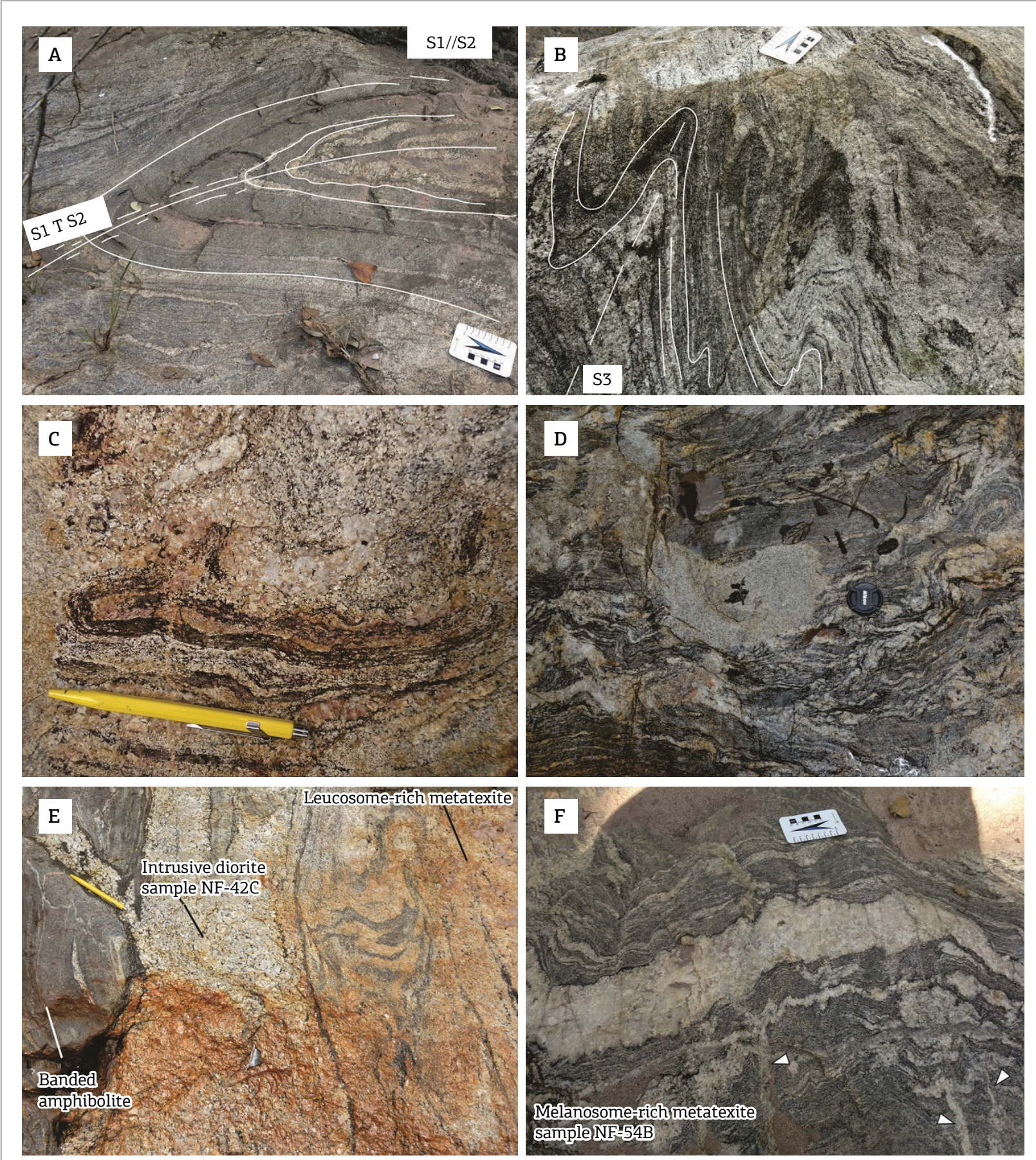

Figure 3. (A) Folded stromatic compositional banding in a residuum-rich metatexite; (B) spaced foliation of the third deformational phase; (C) leucosome-rich metatexite with biotite schlieren showing fold rootless; (D) residuum-rich metatexite with deformed stromatic structure and fine phaneritic granitoid; (E) dioritic injection among amphibolite enclaves and transitional metatexite with schlieren; $(F)$ folded melanosome-rich metatexite with late leucosome. 
occurs as a mineral reaction product. Plagioclase is tabular shaped with albite twinning and locally showing myrmekitic texture. Biotite is interstitial with quartz and sillimanite fringes (Fig. 4E and Fig. 4F). Garnet is relictual xenomorphic and replaced by biotite and plagioclase.

\section{Amphibolite enclaves}

Amphibolite enclaves are melanocratic with nematoblastic texture and can be divided in two types: banded amphibolites and amphibole schists. Banded amphibolites are composed of quartz + hornblende,
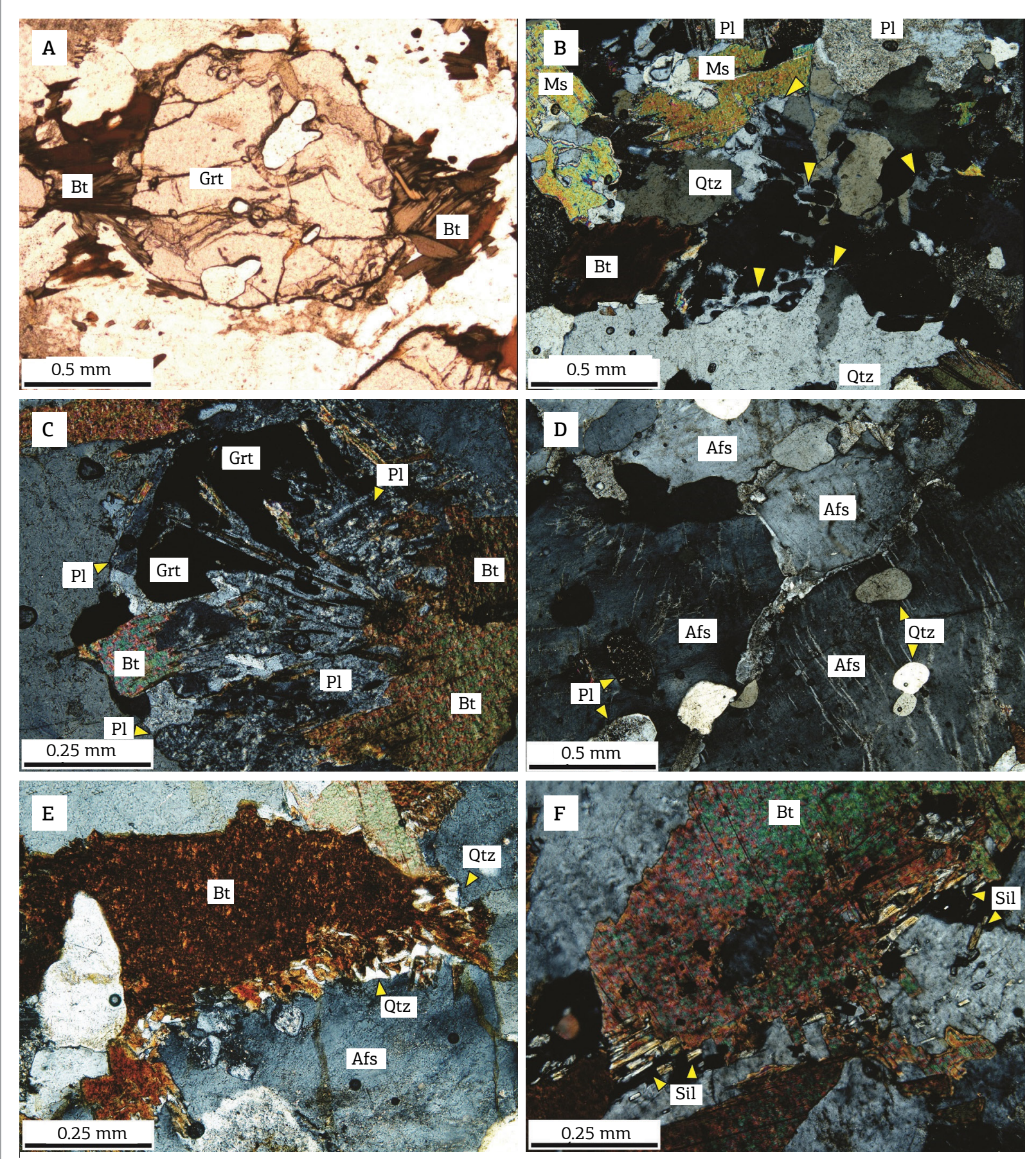

Figure 4. (A) Pre-cinematic garnet with quartz inclusions and biotite strain shadow; (B) pseudomorphic melting drops indicate the enveloping of muscovite and rounded quartz grains; (C) garnet partially pseudomorphed to biotite and plagioclase; (D) rounded plagioclase inclusions within subhedral orthoclase; (E) biotite with quartz intergrowing as fringes; (F) sillimanite indicating high metamorphic grade. Parallel polarization on (A) and cross polarizationon (B, C, D, E and F). Abbreviations are as in Fettes \& Desmons (2008). 
diopside + plagioclase, and plagioclase + hornblende bands (Fig. 5A). Quartz shows sharp borders showing a granoblastic texture (Fig. 5B). Diopside is xenomorphic and has a nematoblastic texture when orientated. When diopside envelops clinozoisite and plagioclase, it forms a poikiloblastic texture (Fig. 5C). Brown hornblende and plagioclase have a nematoblastic texture (Fig. 5D), similar to the diopside + plagioclase band. Biotite, tremolite-actinolite,
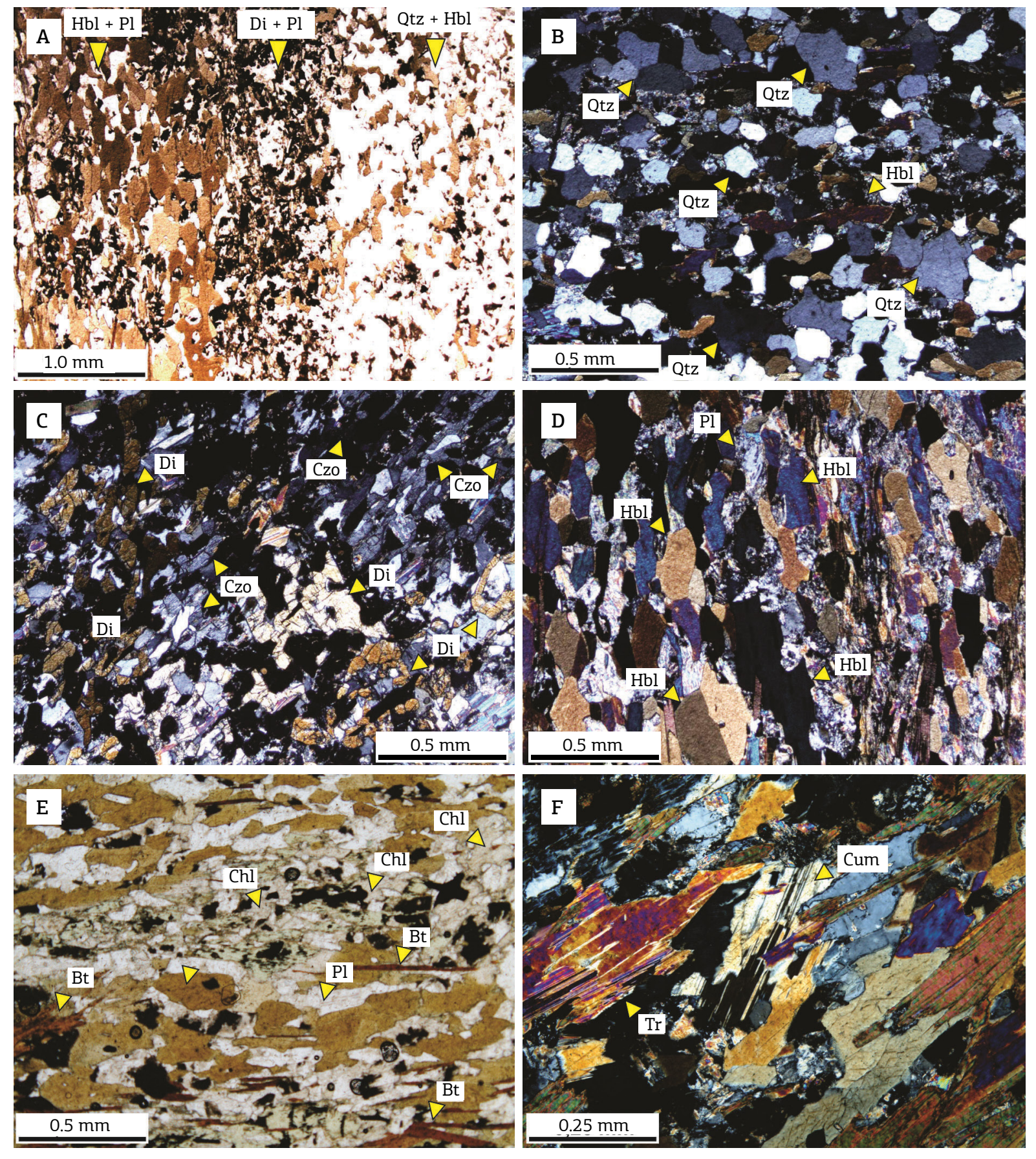

Figure 5. Photomicrographs of banded amphibolite enclaves (A, B, C, and D) and amphibole schist (E and F) showing: (A) compositional grading among hornblende + plagioclase, diopside + plagioclase, and quartz + hornblende bands; (B) detail of a moderate amount of quartz within the quartz + hornblende band; (C) poikiloblast diopside; (D) nematoblastic texture composed of hornblende and pseudomorphosed plagioclase; (E) nematoblastic texture composed of hornblende, quartz, plagioclase, biotite, and chlorite; (F) tremolite/actinolite and cummingtonite. Parallel polarization on A and E and cross polarization on B, C, D and F. 
cummingtonite, epidote and chlorite occur locally as retrometamorphic reaction products.

The amphibole schist occurs as tabular fragments composed essentially of brown hornblende, plagioclase (andesine), quartz and biotite (Fig. 5E). These minerals are orientated and show evidence of retrometamorphism associated to the plagioclase saussuritization, biotite and hornblende chloritization, and are associated to tremolite and cummingtonite (Fig. 5F).

\section{Dioritic injections}

Dioritic injections are leucocratic, equigranular, coarse-grained with megacrysts of plagioclase $(60-73 \%)$, alkali feldspar $(7-15 \%)$, biotite $(10-15 \%)$ and quartz $(<5 \%)$. Garnet $(<2 \%)$, epidote and monazite $(<2 \%)$ are accessory minerals. Chlorite, epidote, calcite and prehnite are secondary minerals. Andesine $(1.5-5 \mathrm{~mm})$ is euhedral to subhedral tabular with albite twinning and a turbid aspect due to saussuritization, sericitization and carbonatization processes. When fine grained, the andesine shows a myrmekite texture
(Fig. 6A). The microcline $(3-5 \mathrm{~mm}$ ) has chessboard twinning (Fig. 6A), tabular habit and drops and string perthites. Biotite $(1-3 \mathrm{~mm})$ occurs sparsely as the main mafic mineral and shows replacement by chlorite, muscovite and prehnite crystallization along the cleavage planes (Fig. 6B). Quartz occurs as interstitial mineral among feldspar megacrystals and shows anhedral form with embayments and inclusions (Fig. $6 \mathrm{C})$. Garnet $(0.1-5 \mathrm{~mm})$ occurs locally as rounded porphyroblasts or as small restitic grains enveloped by plagioclase and biotite aggregates. Chlorite and opaque minerals are commonly found along garnet porphyroblast fractures (Fig. 6D).

\section{GEOCHEMISTRY}

\section{Analytical procedures}

Major, minor and trace element concentrations were determined for 26 samples of the FAM. Raw data are shown on Table 2. Due to the structural complexity and
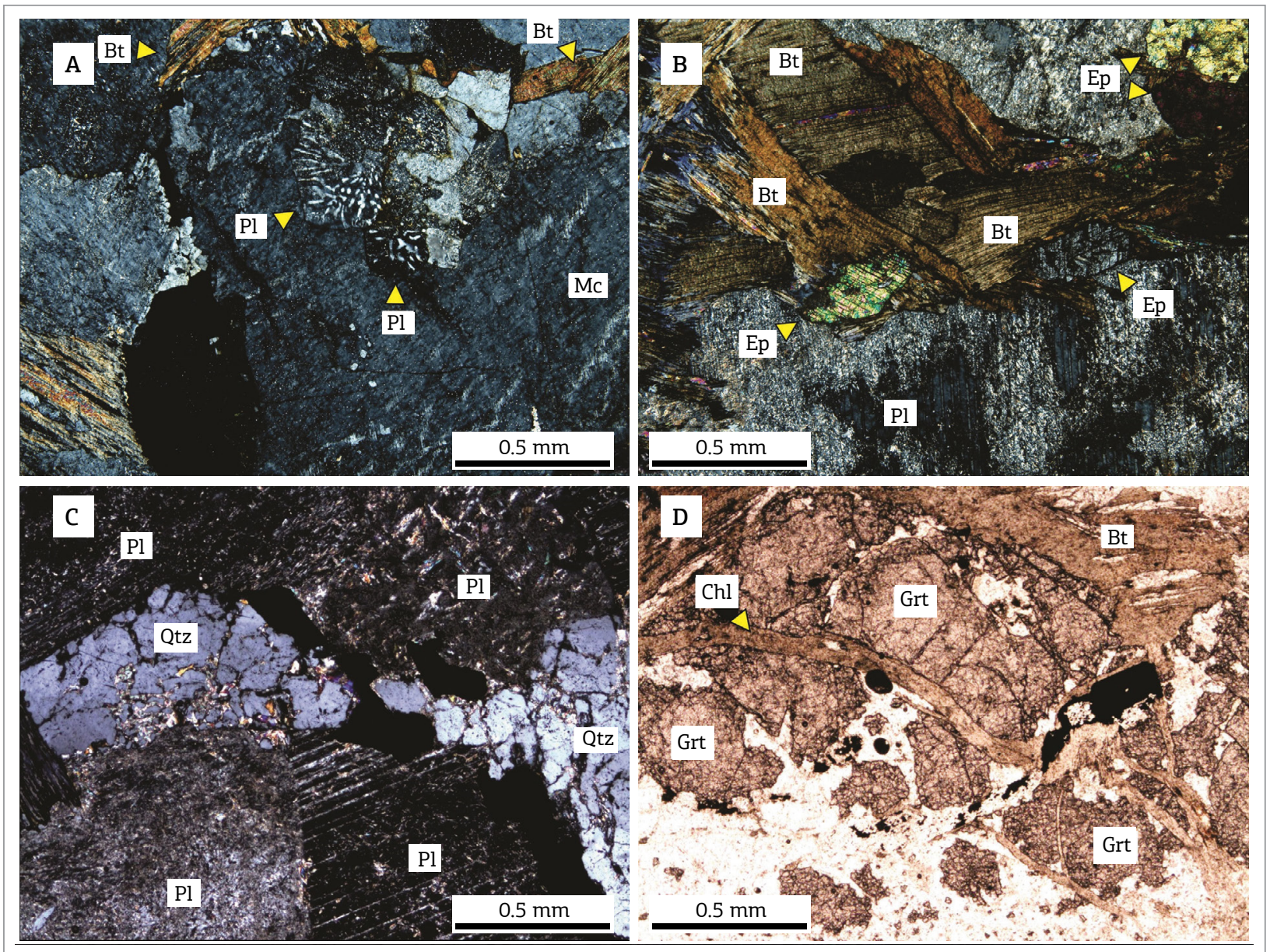

Figure 6. Photomicrographs of dioritic injections showing (A) myrmekitic texture in plagioclase; (B) biotite replaced by chlorite, prehnite, and epidote; $(C)$ fractured quartz with embayments and fractures; (D) fractured garnet associated with chlorite. Parallel polarization on D and cross polarization on A, B, and C. 
Petrogenesis and geochronology of the Furna Azul Migmatite

Table 2. Geochemical data of Furna Azul Migmatite samples (oxides in percentage and trace elements in ppm).

\begin{tabular}{|c|c|c|c|c|c|c|c|c|c|c|c|c|c|}
\hline \multirow{2}{*}{ Sample } & \multicolumn{13}{|c|}{ Transitional metatexite } \\
\hline & ML2 & ML3 & ML1 & NF191 & NF42B5 & MRL2 & NF54 & NF42B & NF42B3 & MRL1 & MRL4 & MRL3 & NF54CH \\
\hline $\mathrm{SiO}_{2}$ & 76.21 & 76.18 & 75.9 & 74.58 & 74.39 & 74.14 & 69.2 & 68.79 & 73.88 & 73.83 & 73.46 & 73.42 & 62.38 \\
\hline $\mathrm{TiO}_{2}$ & 0.19 & 0.21 & 0.19 & 0.26 & 0.53 & 0.4 & 0.57 & 0.64 & 0.38 & 0.42 & 0.38 & 0.39 & 0.74 \\
\hline $\mathrm{Al}_{2} \mathrm{O}_{3}$ & 12.6 & 12.63 & 12.66 & 12.96 & 12.06 & 12.96 & 14.68 & 14.56 & 13.14 & 12.91 & 13.45 & 13.22 & 17.75 \\
\hline $\mathrm{Fe}_{2} \mathrm{O}_{3}$ & 1.2 & 1.24 & 1.23 & 2.8 & 3.91 & 2.52 & 4.16 & 4.81 & 2.65 & 2.7 & 2.51 & 2.62 & 5.66 \\
\hline $\mathrm{MnO}$ & 0.01 & 0.01 & 0.01 & 0.03 & 0.05 & 0.02 & 0.05 & 0.07 & 0.03 & 0.02 & 0.02 & 0.03 & 0.06 \\
\hline $\mathrm{MgO}$ & 0.31 & 0.32 & 0.3 & 0.68 & 1.08 & 0.66 & 1.06 & 1.29 & 0.66 & 0.71 & 0.63 & 0.67 & 1.56 \\
\hline $\mathrm{CaO}$ & 1.41 & 1.41 & 1.43 & 2.97 & 1.86 & 1.74 & 1.93 & 1.76 & 1.45 & 1.7 & 1.74 & 1.74 & 1.41 \\
\hline $\mathrm{Na}_{2} \mathrm{O}$ & 3.3 & 3.27 & 3.35 & 3.29 & 2.84 & 3.69 & 3.61 & 3.08 & 3.2 & 3.64 & 3.73 & 3.67 & 3.28 \\
\hline $\mathrm{K}_{2} \mathrm{O}$ & 3.7 & 3.73 & 3.62 & 0.87 & 2.35 & 2.6 & 3.26 & 3.89 & 3.76 & 2.61 & 2.92 & 2.73 & 6.05 \\
\hline $\mathrm{P}_{2} \mathrm{O}_{5}$ & 0.05 & 0.05 & 0.04 & 0.05 & 0.05 & 0.05 & 0.04 & 0.07 & 0.04 & 0.05 & 0.04 & 0.04 & 0.06 \\
\hline $\mathrm{Cr}_{2} \mathrm{O}_{3}$ & & & & & 0.007 & 0.003 & 0.004 & 0.008 & 0.004 & 0.003 & 0.003 & 0.003 & 0.011 \\
\hline Sum & 99.81 & 99.82 & 99.81 & 99.88 & 99.83 & 99.79 & 99.84 & 99.78 & 99.87 & 99.81 & 99.79 & 99.78 & 99.76 \\
\hline LOI & 0.8 & 0.8 & 1.1 & 1.4 & 0.7 & 1 & 1.3 & 0.8 & 0.7 & 1.2 & 0.9 & 1.3 & 0.8 \\
\hline $\mathrm{Ba}$ & 531 & 530 & 474 & 141 & 625 & 318 & 495 & 839 & 464 & 336 & 344 & 337 & 880 \\
\hline $\mathrm{Rb}$ & 102.1 & 103.6 & 99.1 & 53.7 & 89 & 101.2 & 119.6 & 119 & 121.5 & 106.9 & 106.3 & 105.9 & 207.2 \\
\hline $\mathrm{Sr}$ & 174.2 & 165.3 & 169.2 & 337.2 & 240.8 & 162.1 & 220.9 & 242.8 & 164.4 & 158.7 & 168.2 & 168 & 229.9 \\
\hline $\mathrm{Zr}$ & 174.7 & 184 & 124.2 & 235.8 & 204.5 & 266.4 & 252.1 & 322.4 & 209.4 & 297 & 252.3 & 294.1 & 253.3 \\
\hline $\mathrm{Nb}$ & 6 & 6.4 & 6.9 & 20.6 & 14.2 & 12.8 & 15.1 & 20.3 & 10.5 & 13.3 & 11.8 & 11.9 & 17.8 \\
\hline $\mathrm{Ni}$ & 5.4 & 5.4 & 6.4 & 5.1 & 15.2 & 8.8 & 13.2 & 21.3 & 9 & 9.2 & 8.6 & 10.1 & 31.9 \\
\hline Co & 85.2 & 70.4 & 112.6 & 3.1 & 7.1 & 96.1 & 6 & 7.8 & 4.1 & 68 & 88.4 & 92.2 & 13.3 \\
\hline $\mathrm{Zn}$ & 39 & 40 & 39 & 102 & 82 & 77 & 100 & 92 & 71 & 81 & 82 & 80 & 149 \\
\hline $\mathrm{La}$ & 22 & 23.9 & 21.2 & 34.2 & 35.6 & 37 & 46.5 & 50.5 & 38.5 & 35.6 & 40 & 38.4 & 37.5 \\
\hline $\mathrm{Ce}$ & 45.9 & 49.3 & 37.6 & 73.9 & 75.3 & 78.9 & 102.8 & 114.6 & 80.7 & 74.9 & 85.7 & 82.9 & 78.9 \\
\hline $\operatorname{Pr}$ & 5.24 & 5.86 & 4.21 & 9.19 & 8.86 & 9.39 & 12.23 & 12.89 & 10.1 & 9.05 & 10.24 & 9.82 & 8.92 \\
\hline $\mathrm{Nd}$ & 20.2 & 22.4 & 15.4 & 34.2 & 31.7 & 36.3 & 46.4 & 49.9 & 40.1 & 36.8 & 40.1 & 38.3 & 33.2 \\
\hline Sm & 4.08 & 4.71 & 4.01 & 7.99 & 6.46 & 8.08 & 9.13 & 8.99 & 8.28 & 7.66 & 8.86 & 8.29 & 6.75 \\
\hline $\mathrm{Eu}$ & 0.87 & 0.86 & 0.86 & 0.73 & 1.21 & 0.91 & 1.16 & 1.44 & 0.97 & 0.81 & 0.86 & 0.85 & 1.29 \\
\hline $\mathrm{Gd}$ & 3.94 & 4.24 & 3.84 & 9.18 & 5.5 & 7.59 & 7.49 & 7.85 & 8.26 & 7.36 & 8.23 & 7.78 & 7.05 \\
\hline $\mathrm{Tb}$ & 0.58 & 0.59 & 0.58 & 1.83 & 0.87 & 1.24 & 0.86 & 1.24 & 1.38 & 1.29 & 1.32 & 1.27 & 1.21 \\
\hline Dy & 2.88 & 2.61 & 3.3 & 11.87 & 5.95 & 6.94 & 3.34 & 7.32 & 7.67 & 7.43 & 7.63 & 7.52 & 7.68 \\
\hline Ho & 0.45 & 0.41 & 0.62 & 2.72 & 1.48 & 1.24 & 0.41 & 1.92 & 1.61 & 1.38 & 1.41 & 1.41 & 1.41 \\
\hline Er & 1.3 & 1.12 & 1.78 & 7.9 & 4.67 & 3.76 & 1.18 & 5.97 & 4.23 & 4.17 & 4.23 & 4.34 & 3.88 \\
\hline $\mathrm{Tm}$ & 0.19 & 0.15 & 0.27 & 1.3 & 0.77 & 0.55 & 0.16 & 1.07 & 0.61 & 0.64 & 0.64 & 0.64 & 0.62 \\
\hline $\mathrm{Yb}$ & 1.1 & 0.98 & 1.83 & 8.41 & 4.61 & 3.39 & 1.04 & 7.16 & 4.04 & 3.91 & 3.94 & 4.14 & 3.99 \\
\hline $\mathrm{Lu}$ & 0.18 & 0.16 & 0.28 & 1.19 & 0.7 & 0.56 & 0.18 & 1.09 & 0.52 & 0.65 & 0.61 & 0.61 & 0.56 \\
\hline $\mathrm{Y}$ & 13.1 & 11 & 17.3 & 73.6 & 38.7 & 36.5 & 14.2 & 51 & 44.1 & 40.6 & 37.7 & 40.1 & 40.5 \\
\hline Cs & 0.6 & 0.7 & 0.7 & 1.9 & 1.3 & 1.2 & 1.5 & 1.7 & 1.2 & 1.5 & 1.2 & 1.2 & 2.7 \\
\hline $\mathrm{Ta}$ & 0.4 & 0.4 & 0.5 & 1.8 & 0.8 & 0.7 & 0.5 & 1.1 & 0.5 & 0.6 & 0.7 & 0.6 & 0.9 \\
\hline $\mathrm{Hf}$ & 6.1 & 6 & 4.5 & 8.8 & 6.5 & 8.8 & 9.1 & 8.7 & 7.5 & 9.9 & 8.3 & 9.7 & 7.5 \\
\hline $\mathrm{U}$ & 1.7 & 1.6 & 1.5 & 4.7 & 1.9 & 2.2 & 2.2 & 3 & 2.2 & 2.7 & 2.6 & 2.8 & 2 \\
\hline V & 17 & 24 & 16 & 21 & 52 & 36 & 60 & 58 & 28 & 40 & 36 & 34 & 78 \\
\hline $\mathrm{W}$ & 501.5 & 394.1 & 618.7 & & 0.5 & 537.8 & & 0.6 & 0.6 & 367.9 & 490.4 & 479.3 & \\
\hline Mo & 0.3 & 0.2 & 0.4 & 0.6 & 0.7 & 0.2 & 1.5 & 0.7 & 0.3 & 0.3 & 0.2 & 0.2 & 2 \\
\hline $\mathrm{Cu}$ & 2.7 & 2.8 & 2.7 & 3.9 & 6.4 & 4.6 & 2.5 & 9 & 3.1 & 4.5 & 5.4 & 5.1 & 21.4 \\
\hline $\mathrm{Pb}$ & 9.2 & 8.8 & 10 & 8.6 & 7.1 & 8.7 & 10.7 & 8.3 & 8.5 & 7.5 & 8.8 & 8.9 & 8.3 \\
\hline $\mathrm{Ga}$ & 15.1 & 16.2 & 15.8 & 23.3 & 16.7 & 18.4 & 21.9 & 19 & 16.4 & 19 & 19.5 & 18.7 & 27 \\
\hline Th & 6.7 & 7.5 & 6.4 & 9.1 & 9.5 & 12.5 & 11.6 & 16.1 & 12.7 & 11.6 & 12.9 & 12.5 & 9.5 \\
\hline $\mathrm{Rb} / \mathrm{Ba}$ & 0.19 & 0.20 & 0.21 & 0.38 & 0.14 & 0.32 & 0.24 & 0.14 & 0.26 & 0.32 & 0.31 & 0.31 & 0.24 \\
\hline $\mathrm{Rb} / \mathrm{Sr}$ & 0.59 & 0.63 & 0.59 & 0.16 & 0.37 & 0.62 & 0.54 & 0.49 & 0.74 & 0.67 & 0.63 & 0.63 & 0.90 \\
\hline $\mathrm{FeOt}$ & 1.08 & 1.12 & 1.11 & 2.52 & 3.52 & 2.27 & 3.74 & 4.33 & 2.38 & 2.43 & 2.26 & 2.36 & 5.09 \\
\hline $\mathrm{mg}^{*}$ & 33.85 & 33.83 & 32.58 & 32.48 & 35.37 & 34.16 & 33.55 & 34.70 & 33.04 & 34.25 & 33.21 & 33.63 & 35.32 \\
\hline $\mathrm{A} / \mathrm{NK}$ & 1.34 & 1.34 & 1.34 & 2.04 & 1.67 & 1.46 & 1.55 & 1.57 & 1.41 & 1.46 & 1.45 & 1.47 & 1.49 \\
\hline $\mathrm{A} / \mathrm{CNK}$ & 1.05 & 1.05 & 1.05 & 1.10 & 1.14 & 1.08 & 1.13 & 1.17 & 1.10 & 1.08 & 1.08 & 1.09 & 1.22 \\
\hline $\mathrm{K}_{2} \mathrm{O} / \mathrm{Na}_{2} \mathrm{O}$ & 1.12 & 1.14 & 1.08 & 0.26 & 0.83 & 0.70 & 0.90 & 1.26 & 1.18 & 0.72 & 0.78 & 0.74 & 1.84 \\
\hline
\end{tabular}


Table 2. Continuation.

\begin{tabular}{|c|c|c|c|c|c|c|c|c|c|c|c|c|c|}
\hline \multirow{2}{*}{ Sample } & \multicolumn{7}{|c|}{ Residuum-rich metatexite } & \multicolumn{6}{|c|}{$\begin{array}{l}\text { Diorite injection } \\
\end{array}$} \\
\hline & MRR3 & NF54B & MRR2 & MRR4 & NF54B2 & NF54B1 & MRR1 & NF42C4 & $\mathrm{NF} 42 \mathrm{C} 2$ & DIO1 & DIO2 & DIO3 & NF42C3 \\
\hline $\mathrm{SiO}_{2}$ & 70.08 & 69.89 & 69.79 & 69.71 & 69.69 & 69.6 & 69.54 & 58.15 & 57.23 & 56.03 & 55.77 & 54.8 & 52.96 \\
\hline $\mathrm{TiO}_{2}$ & 0.75 & 0.72 & 0.74 & 0.76 & 0.74 & 0.77 & 0.75 & 0.66 & 0.94 & 1.05 & 0.97 & 1.12 & 1.62 \\
\hline $\mathrm{Al}_{2} \mathrm{O}_{3}$ & 13.72 & 13.95 & 13.93 & 13.76 & 13.78 & 14.02 & 13.78 & 21.57 & 20.99 & 21.36 & 21.56 & 21.42 & 20.49 \\
\hline $\mathrm{Fe}_{2} \mathrm{O}_{3}$ & 5.34 & 5.71 & 5.54 & 5.49 & 5.83 & 5.7 & 5.38 & 3.34 & 4.61 & 4.7 & 4.56 & 5.03 & 7.48 \\
\hline $\mathrm{MnO}$ & 0.08 & 0.08 & 0.09 & 0.08 & 0.08 & 0.1 & 0.1 & 0.02 & 0.03 & 0.03 & 0.03 & 0.03 & 0.05 \\
\hline $\mathrm{MgO}$ & 1.57 & 1.58 & 1.6 & 1.6 & 1.6 & 1.56 & 1.53 & 1.07 & 1.54 & 1.6 & 1.57 & 1.69 & 2.49 \\
\hline $\mathrm{CaO}$ & 2.75 & 2.58 & 2.67 & 2.64 & 2.58 & 2.75 & 2.77 & 4.2 & 4.01 & 4.23 & 4.3 & 4.36 & 4.58 \\
\hline $\mathrm{Na}_{2} \mathrm{O}$ & 3.03 & 3.21 & 3.03 & 3.09 & 3.18 & 3.1 & 3.03 & 6.54 & 6 & 6.13 & 6.35 & 6.07 & 5.04 \\
\hline $\mathrm{K}_{2} \mathrm{O}$ & 1.43 & 1.57 & 1.53 & 1.5 & 1.65 & 1.43 & 1.41 & 2 & 2.37 & 2.41 & 2.23 & 2.35 & 2.3 \\
\hline $\mathrm{P}_{2} \mathrm{O}_{5}$ & 0.14 & 0.14 & 0.13 & 0.13 & 0.19 & 0.19 & 0.13 & 0.25 & 0.32 & 0.22 & 0.24 & 0.22 & 0.18 \\
\hline $\mathrm{Cr}_{2} \mathrm{O}_{3}$ & 0.008 & 0.01 & 0.008 & 0.008 & 0.007 & 0.007 & 0.007 & 0.002 & 0.003 & & & 0.002 & 0.004 \\
\hline Sum & 99.76 & 99.86 & 99.77 & 99.77 & 99.86 & 99.83 & 99.77 & 99.44 & 99.26 & 99.43 & 99.43 & 99.43 & 99.49 \\
\hline LOI & 0.9 & 0.4 & 0.7 & 1 & 0.5 & 0.6 & 1.3 & 1.6 & 1.2 & 1.7 & 1.8 & 2.3 & 2.3 \\
\hline $\mathrm{Ba}$ & 306 & 278 & 297 & 289 & 294 & 326 & 288 & 229 & 255 & 289 & 250 & 267 & 339 \\
\hline $\mathrm{Rb}$ & 81.6 & 85.2 & 88 & 85.7 & 91.8 & 83.6 & 81.6 & 102.6 & 125.3 & 110.4 & 104.8 & 114.9 & 100.8 \\
\hline $\mathrm{Sr}$ & 227.9 & 242.7 & 219 & 219.3 & 238.5 & 245.7 & 226.3 & 379.3 & 358.7 & 311.8 & 326.7 & 330.1 & 312.5 \\
\hline $\mathrm{Zr}$ & 235.9 & 223.2 & 249.9 & 277.9 & 245 & 288.6 & 261.3 & 22 & 18.9 & 22.8 & 24.7 & 20.6 & 15.7 \\
\hline $\mathrm{Nb}$ & 12.4 & 11.5 & 13.5 & 13 & 12 & 11.9 & 13.2 & 17.2 & 23.7 & 27.6 & 25.2 & 28.8 & 40.3 \\
\hline $\mathrm{Ni}$ & 21.2 & 25.8 & 23.4 & 19.9 & 26 & 23.6 & 20.6 & 12.2 & 14.7 & 13.9 & 14.5 & 15 & 22.6 \\
\hline Co & 93.1 & 12.8 & 82.3 & 92.3 & 12.5 & 11.9 & 108.9 & 5.9 & 8.6 & 33.6 & 25.4 & 30.5 & 13.2 \\
\hline $\mathrm{Zn}$ & 87 & 93 & 96 & 90 & 100 & 97 & 90 & 89 & 123 & 118 & 116 & 122 & 207 \\
\hline $\mathrm{La}$ & 40.6 & 38 & 44.5 & 41.8 & 42.2 & 46.4 & 41.4 & 640.4 & 871 & 606.4 & 609.3 & 595.8 & 489.3 \\
\hline $\mathrm{Ce}$ & 82.4 & 79.9 & 84.7 & 85.1 & 86.2 & 97.4 & 82.5 & 1547.4 & 2088.5 & 1361.8 & 1417.1 & 1395.2 & 1156.4 \\
\hline $\mathrm{Pr}$ & 9.43 & 9.65 & 9.94 & 9.89 & 10.71 & 11.52 & 9.67 & 196.6 & 271.15 & 172.69 & 176.5 & 179.31 & 146.99 \\
\hline $\mathrm{Nd}$ & 36.4 & 36.4 & 38.5 & 37.5 & 39.9 & 46.9 & 37.5 & 795 & 1085 & 701 & 710.5 & 714.2 & 578.1 \\
\hline $\mathrm{Sm}$ & 7.24 & 6.76 & 7.81 & 7.58 & 7.8 & 8.61 & 7.28 & 163.41 & 223.24 & 145.2 & 148.36 & 150.02 & 119.27 \\
\hline $\mathrm{Eu}$ & 1.11 & 1.25 & 1.15 & 1.18 & 1.34 & 1.31 & 1.17 & 3.18 & 3.74 & 2.91 & 2.91 & 2.88 & 2.51 \\
\hline $\mathrm{Gd}$ & 6.82 & 6.58 & 7.29 & 6.97 & 7.45 & 7.87 & 6.78 & 137.35 & 183.48 & 116.43 & 119.76 & 121.82 & 97.45 \\
\hline $\mathrm{Tb}$ & 1.13 & 1.08 & 1.27 & 1.24 & 1.27 & 1.3 & 1.15 & 15.16 & 20.05 & 13.53 & 14.03 & 14.4 & 10.77 \\
\hline Dy & 6.73 & 5.55 & 7.57 & 7.62 & 6.94 & 7.61 & 7.19 & 55.75 & 76.24 & 49.91 & 51.48 & 53.22 & 40.39 \\
\hline Ho & 1.28 & 1.25 & 1.43 & 1.41 & 1.55 & 1.42 & 1.4 & 6.05 & 8.83 & 5.17 & 5.67 & 5.64 & 4.6 \\
\hline Er & 3.52 & 3.67 & 3.93 & 3.98 & 4.2 & 4.21 & 3.87 & 8.94 & 14.15 & 8.3 & 8.7 & 9.11 & 6.92 \\
\hline $\mathrm{Tm}$ & 0.54 & 0.58 & 0.63 & 0.6 & 0.63 & 0.72 & 0.59 & 0.79 & 1.21 & 0.69 & 0.76 & 0.79 & 0.62 \\
\hline $\mathrm{Yb}$ & 3.36 & 3.72 & 3.78 & 3.8 & 3.84 & 4.07 & 3.63 & 4.18 & 6.24 & 3.46 & 3.8 & 3.85 & 3.34 \\
\hline $\mathrm{Lu}$ & 0.52 & 0.56 & 0.6 & 0.56 & 0.61 & 0.65 & 0.57 & 0.42 & 0.68 & 0.36 & 0.39 & 0.42 & 0.34 \\
\hline $\mathrm{Y}$ & 36.2 & 34.8 & 40.8 & 38.4 & 40.8 & 42.7 & 38.6 & 161.2 & 236.5 & 140.8 & 153.4 & 153.7 & 121.7 \\
\hline Cs & 1.7 & 1.3 & 1.7 & 1.6 & 1.9 & 1.9 & 1.4 & 1.9 & 2.3 & 1.6 & 1.6 & 1.7 & 2.1 \\
\hline $\mathrm{Ta}$ & 1 & 0.7 & 1 & 1.2 & 0.7 & 0.8 & 1.1 & 0.8 & 0.9 & 0.9 & 0.8 & 1.2 & 1.5 \\
\hline $\mathrm{Hf}$ & 7.2 & 7.1 & 7 & 7.5 & 6.8 & 7.2 & 7.1 & 0.5 & 1 & 0.7 & 0.7 & 0.6 & 0.8 \\
\hline $\mathrm{U}$ & 3.2 & 2.8 & 3.3 & 3.3 & 3.2 & 3.8 & 3.2 & 12.3 & 17 & 12 & 12.4 & 12.8 & 9.2 \\
\hline $\mathrm{V}$ & 84 & 62 & 78 & 75 & 68 & 70 & 76 & 59 & 84 & 93 & 89 & 98 & 142 \\
\hline W & 480.9 & 0.7 & 429.8 & 469.1 & & & 561.6 & & & 124.2 & 88.2 & 105.1 & 0.5 \\
\hline Mo & 0.2 & 0.9 & 0.3 & 0.2 & 0.4 & 0.6 & 0.2 & 0.8 & 0.7 & 0.6 & 0.3 & 1.1 & 2.9 \\
\hline $\mathrm{Cu}$ & 6.7 & 11.4 & 7.3 & 5.8 & 11 & 9.4 & 6.9 & 3.1 & 2.9 & 1 & 1 & 0.9 & 2 \\
\hline $\mathrm{Pb}$ & 5.8 & 5.8 & 5.8 & 5.6 & 5.2 & 5.9 & 6.1 & 35.5 & 28.4 & 29.7 & 30.2 & 32.9 & 25.1 \\
\hline $\mathrm{Ga}$ & 18.3 & 16.3 & 20 & 18.1 & 17.6 & 17.6 & 20.7 & 33 & 35.3 & 33 & 33.1 & 34.5 & 35.4 \\
\hline Th & 10.2 & 9.1 & 9.7 & 10.1 & 10.3 & 11.4 & 9.6 & 314.1 & 421.8 & 283.3 & 299.2 & 293.2 & 225.2 \\
\hline $\mathrm{Rb} / \mathrm{Ba}$ & 0.27 & 0.31 & 0.30 & 0.30 & 0.31 & 0.26 & 0.28 & 0.45 & 0.49 & 0.38 & 0.42 & 0.43 & 0.30 \\
\hline $\mathrm{Rb} / \mathrm{Sr}$ & 0.36 & 0.35 & 0.40 & 0.39 & 0.38 & 0.34 & 0.36 & 0.27 & 0.35 & 0.35 & 0.32 & 0.35 & 0.32 \\
\hline $\mathrm{FeOt}$ & 4.80 & 5.14 & 4.98 & 4.94 & 5.25 & 5.13 & 4.84 & 3.01 & 4.15 & 4.23 & 4.10 & 4.53 & 6.73 \\
\hline $\mathrm{mg}^{*}$ & 36.81 & 35.41 & 36.39 & 36.60 & 35.22 & 35.16 & 36.04 & 38.83 & 39.82 & 40.28 & 40.55 & 39.96 & 39.74 \\
\hline $\mathrm{A} / \mathrm{NK}$ & 2.10 & 2.00 & 2.10 & 2.05 & 1.96 & 2.11 & 2.12 & 1.67 & 1.69 & 1.68 & 1.68 & 1.71 & 1.90 \\
\hline $\mathrm{A} / \mathrm{CNK}$ & 1.19 & 1.20 & 1.21 & 1.20 & 1.18 & 1.20 & 1.19 & 1.05 & 1.06 & 1.05 & 1.04 & 1.05 & 1.07 \\
\hline $\mathrm{K}_{2} \mathrm{O} / \mathrm{Na}_{2} \mathrm{O}$ & 0.47 & 0.49 & 0.50 & 0.49 & 0.52 & 0.46 & 0.47 & 0.31 & 0.40 & 0.39 & 0.35 & 0.39 & 0.46 \\
\hline
\end{tabular}


variations of neosome proportion, the analyzed samples do not necessarily represent individual portions of a migmatite. However, the residuum metatexites showed a compositional pattern that might matches partial melting fractionation. On the other hand, a typical differentiation pattern can be observed in the transitional leucosome-rich metatexite samples.

Around $1 \mathrm{~kg}$ of each sample was crushed, homogenized and pulverized at laboratories of the Universidade Federal do Mato Grosso, Brazil, and sent to Acme Analytical Laboratories for the determination of major and trace elements by inductively coupled plasma-atomic emission spectrometer (ICP-AES) and inductively coupled plasma mass spectrometry (ICP-MS), respectively. Detailed experimental procedures are available online on the Acme homepage. Geochemical data processing was performed using the GeoChemical Data toolkit (GCDkit) software by Janoušek et al. (2011).

\section{Major and trace elements}

Since they display complex structural pattern and distinctive neosome proportions, FAM metatexites are considered composite rocks. The geochemical pattern tends to show progressive melting fractionation characteristics. However, classification diagrams should represent the protholith chemical composition.

In the TAS diagram (Fig. 7A), residuum-rich rocks show granodioritic composition while leucosome tends to granite field. Both are subalkaline. Dioritic injections plot in the syenodiorite field of the alkaline series. All lithotypes plot in the non-tholeiitic and peraluminous fields in the AFM and $\mathrm{A} / \mathrm{NK}$ versus $\mathrm{A} / \mathrm{CNK}$ diagrams, respectively (Fig. $7 \mathrm{~B}$ and Fig. 7C). The tectonic discriminant diagrams, according to Frost et al. (2001), indicate a cordilleran character for the migmatites with a peraluminous affinity for the leucosome (Fig. 7D and Fig. 7E). Dioritic injections are ferroan and plot within the field with alkalic affinity. Metatexites plot in the volcanic arc granite field, and Dioritic injections plot in the syn-collisional field, in the Rb/30-Hf-3Ta ternary diagram (Fig. 7F).

In the Harker (1909) diagrams, all samples show a negative correlation of $\mathrm{TiO}_{2}, \mathrm{Al}_{2} \mathrm{O}_{3}$ and $\mathrm{MgO}$ against $\mathrm{SiO}_{2}, \mathrm{CaO}$, $\mathrm{Na}_{2} \mathrm{O}, \mathrm{K}_{2} \mathrm{O}, \mathrm{FeO}_{\mathrm{t}}$ and $\mathrm{P}_{2} \mathrm{O}_{5}$ (Fig. 8), allowing the recognition of two distinct patterns that correspond to dioritic injections and metatexites. Negative correlations suggest fractionation of mafic minerals and calcic plagioclase. $\mathrm{Al}_{2} \mathrm{O}_{3}, \mathrm{P}_{2} \mathrm{O}_{5}$, and $\mathrm{Na}_{2} \mathrm{O}$ increments could be related to a magmatic evolution from alkaline basalts.

The large ion lithophile elements LILE ( $\mathrm{Ba}, \mathrm{Rb}, \mathrm{Sr}$ and $\mathrm{K}$ ) against $\mathrm{SiO}_{2}$ diagrams (Fig. 9), as observed for major oxides, enable the individualization of dioritic injections and metatexites. Residuum-rich rocks show lower contents of $\mathrm{Ba}, \mathrm{Rb}$, and $\mathrm{K}$, suggesting fractionation processes. Dioritic injection samples show a positive correlation of $\mathrm{Sr}, \mathrm{La}, \mathrm{Eu}, \mathrm{Y}$ and $\mathrm{Yb}$ against $\mathrm{SiO}_{2}$, attesting light rare earth element (LREE) and heavy rare earth element (HREE) enrichment. The negative correlation between $\mathrm{Ba}$ and $\mathrm{K}$ suggests an evolution from intermediate to acidic magmas, which is not the case for rocks with alkaline derivation.

Sawyer (2010) demonstrates that migmatites from Opatica Subprovince, Canada, might have been originated from partial melting of a leucogranodiorite. In the $\mathrm{K}_{2} \mathrm{O}$ versus $\mathrm{CaO}+\mathrm{Na}_{2} \mathrm{O}$ diagram (Fig. 10A), effects of anatexis can be observed: $\mathrm{K}_{2} \mathrm{O}$ enrichment in leucosome-rich metatexites and $\mathrm{CaO}+\mathrm{Na}_{2} \mathrm{O}$ enrichment in residuum-rich rocks. However, FAM metatexites do not overlap with melt fields from granodioritic protholiths, and, yet, they overlap with glass from tonalitic melts, according to experimental works by Castro (2004) and Watkins et al. (2007).

Patiño Douce (1999) features the geochemical patterns of melts from several sources. In Figure 10B and Figure 10C, dioritic injection and residuum-rich metatexite samples match melts from an amphibolite source, while transitional leucosome-rich metatexites match melts from mafic pelites, grauvaques, or calc-alkaline granite.

In the $\mathrm{ACNK}$ versus $\mathrm{SiO}_{2}$ diagram (Fig. 10D), the peraluminous character of the FAM sample is expressed. Sylvester (1998) differentiates collisional peraluminous granites of high-pressure belts (Himalayan) from those of high temperature belts (Lachlan Fold Belt) and uses $\mathrm{Rb} / \mathrm{Ba}$ versus $\mathrm{Rb} / \mathrm{Sr}$ ratios to discriminate $\mathrm{Al}_{2} \mathrm{O}_{3}$-rich (pelites) and $\mathrm{Al}_{2} \mathrm{O}_{3}$-poor (graywackes and psammites) protholiths. In this diagram, the FAM rock ratios suggest derivation from a graywacke protholith (Fig. 10E). CaO/Na 2 O versus $\mathrm{Al}_{2} \mathrm{O}_{3} / \mathrm{TiO}_{2}$ ratios indicate similarities with Lachlan Fold Belt peraluminous granites (Fig. 10F).

\section{Rare earth elements}

Using the primitive-mantle-normalized values of McDonough \& Sun (1995), migmatites show LREE enrichment with Eu depletion, indicating plagioclase fractionation. One sample of leucosome-rich metatexite had low HREE values, suggesting that these elements had an immobile behavior during the melting or that the melting condition did not reach the solubility of these elements. Dioritic injection patterns show intense light and heavy rare earth elements (REE) enrichment, indicating an enriched-mantle source or intense processes of crustal assimilation. Eu depletion suggests plagioclase fractionation (Fig. 11A). 
A

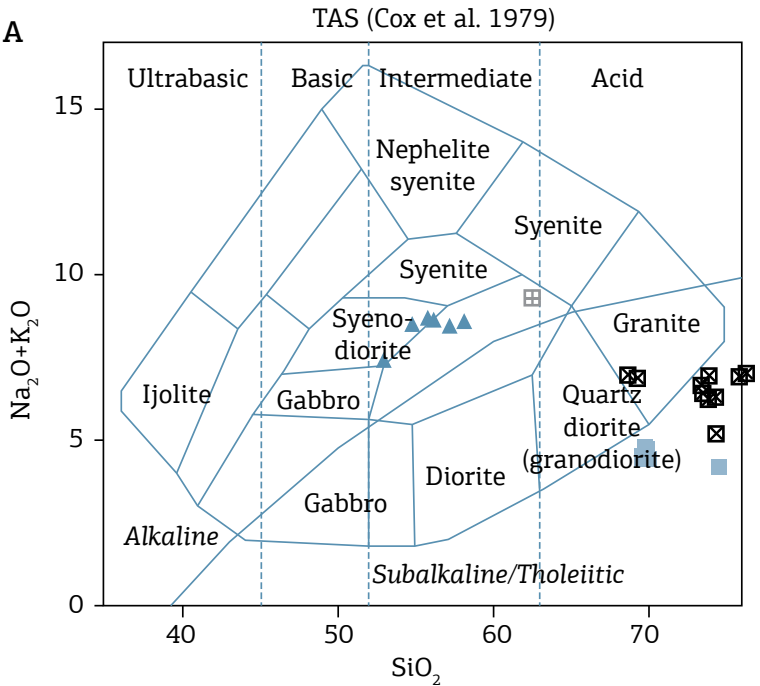

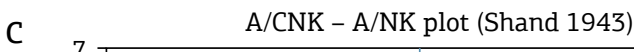

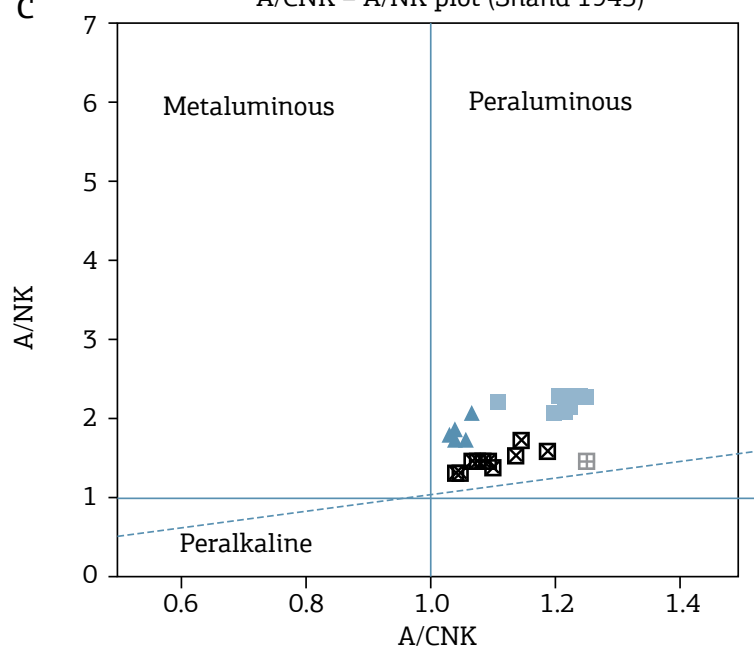

E

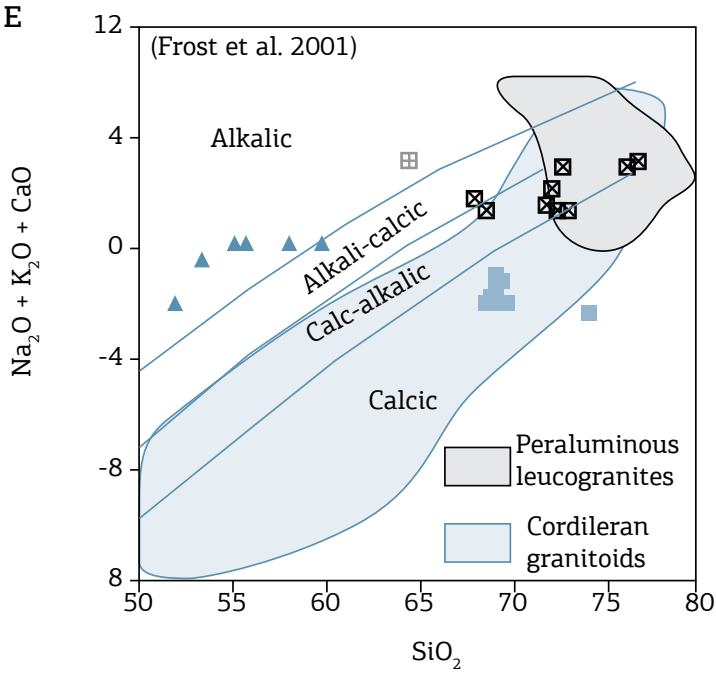

B

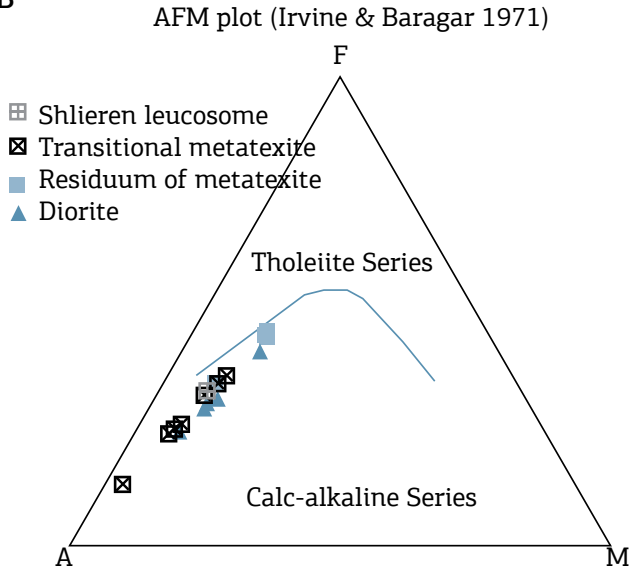

D

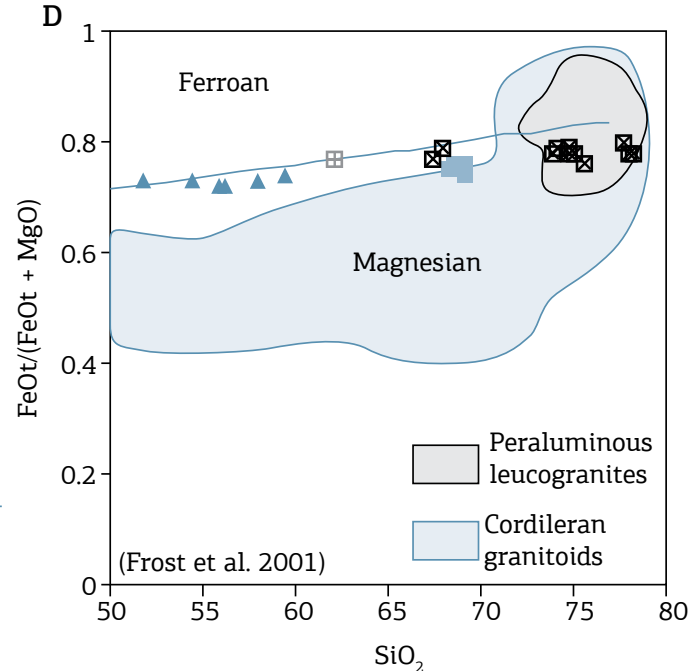

F

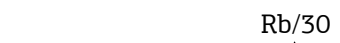

Figure 7. Classification and discriminant diagrams: (A) TAS diagram; (B) AFM diagram; (C) alumina saturation diagram; (D and E) Frost et al. (2001) diagrams; (F) Rb-Hf-Ta ternary diagram (Harris et al. 1986). 
Using the bulk continental crust normalization pattern by Taylor \& McLennan (1995), metatexites show an enriched pattern with negative anomalies for $\mathrm{Sr}$ and $\mathrm{Ti}$ (Fig. 11B), indicating plagioclase, titanite and/or magnetite fractionation or low melting temperatures. The dioritic injection pattern shows LREE, Th and $U$ enrichment, suggesting monazite concentration, and negative anomalies for $\mathrm{Zr}$ and $\mathrm{Hf}$ that might indicate a mantle affinity.
The melanosome-rich and leucosome-rich metatexite patterns, when normalized to the residuum rock average, show positive anomalies for $\mathrm{Ba}$ and $\mathrm{K}$, suggesting that the $\mathrm{k}$-feldspar concentration and $\mathrm{Rb}$ and $\mathrm{Nb}$ values are controlled by the biotite concentration in schlieren (Fig. 11C). The less incompatible element pattern can be indicated by zircon and garnet concentration in residual rocks, due to the recurrence of garnet as a peritectic phase in leucosome.
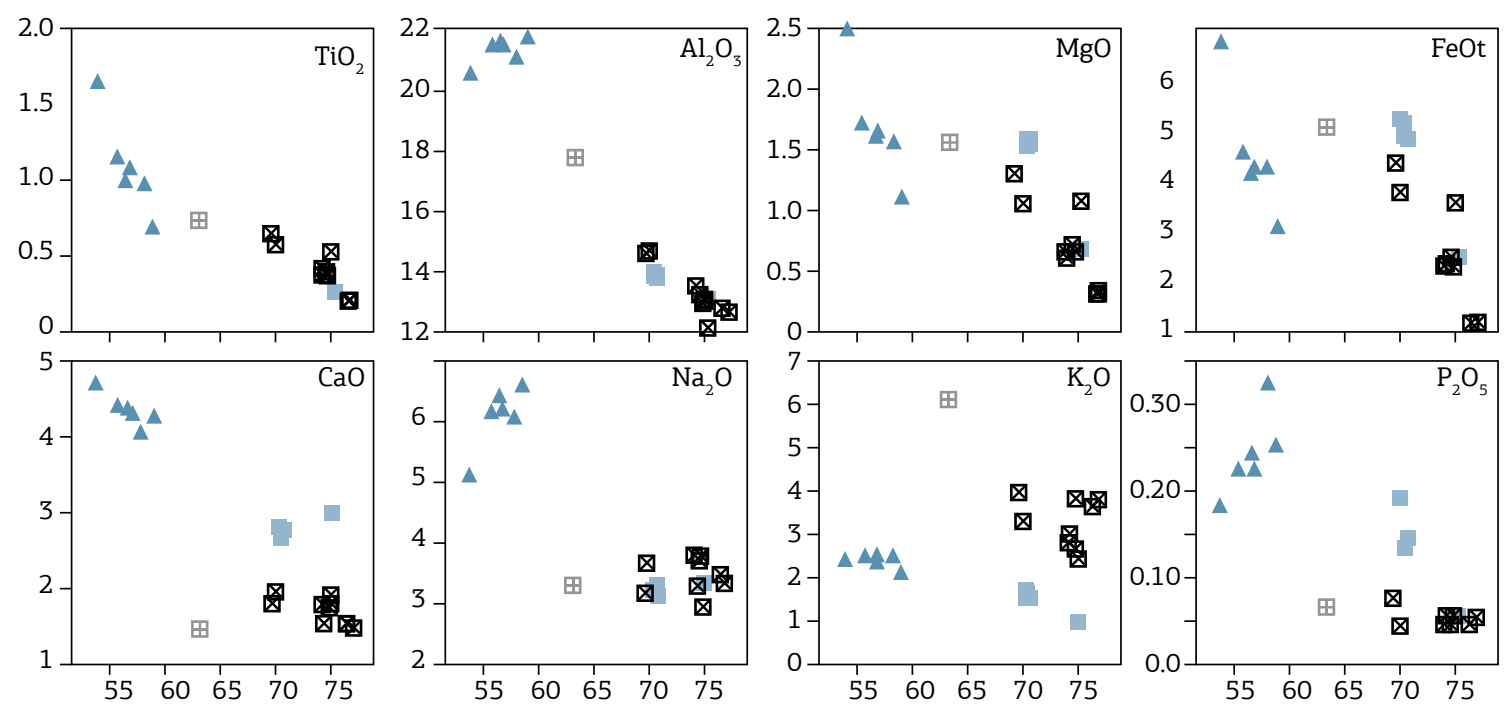

$\Delta$ Diorite Residuum of metatexite $\boldsymbol{\nabla}$ Transitional metatexite $\boxplus$ Schlieren leucosome

Figure 8. Harker (1909) diagrams showing $\mathrm{SiO}_{2}$ versus major element patterns for Furna Azul Migmatite samples.

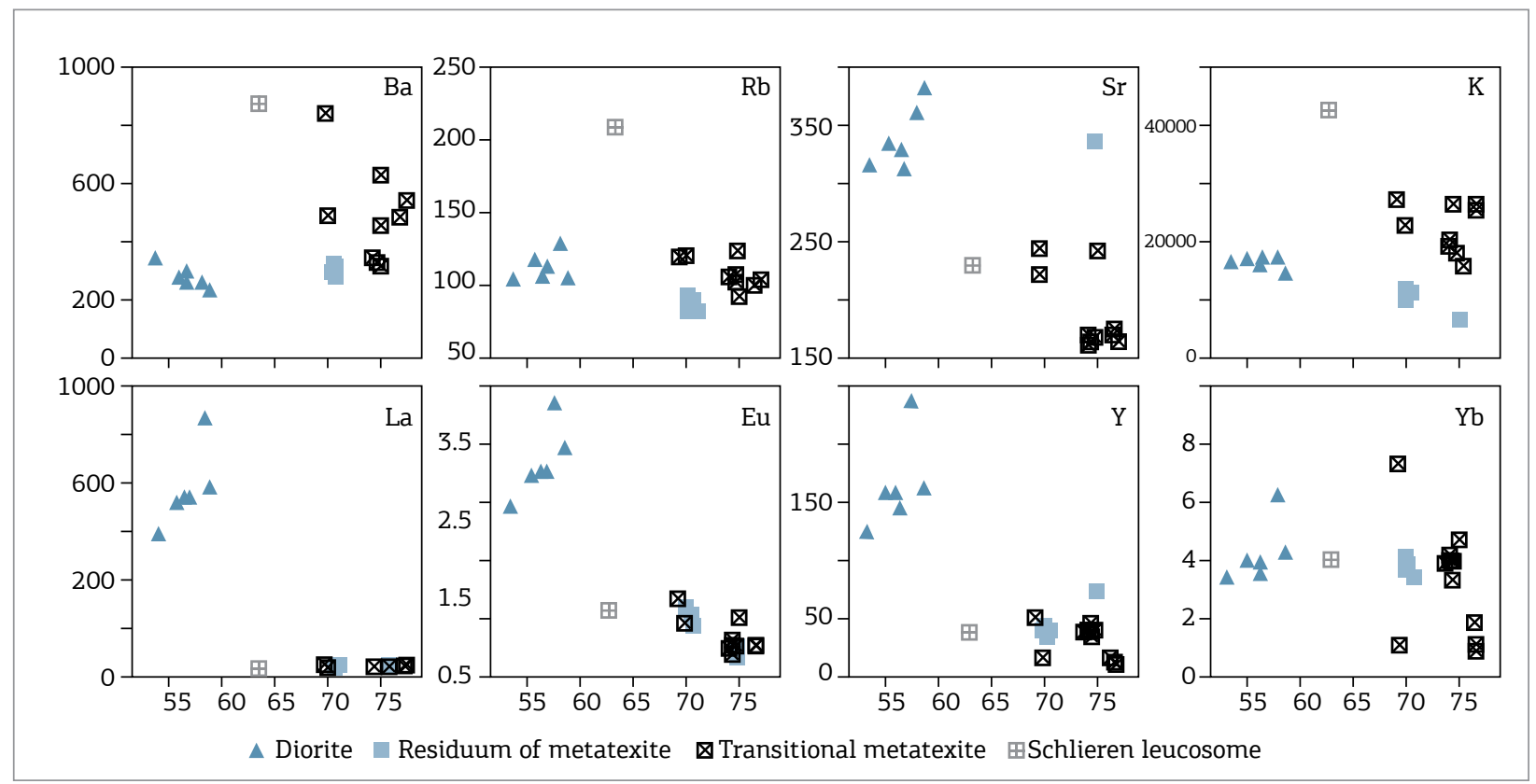

Figure 9. Harker (1909) diagrams showing $\mathrm{SiO}_{2}$ versus minor and trace element patterns for Furna Azul Migmatite samples. 

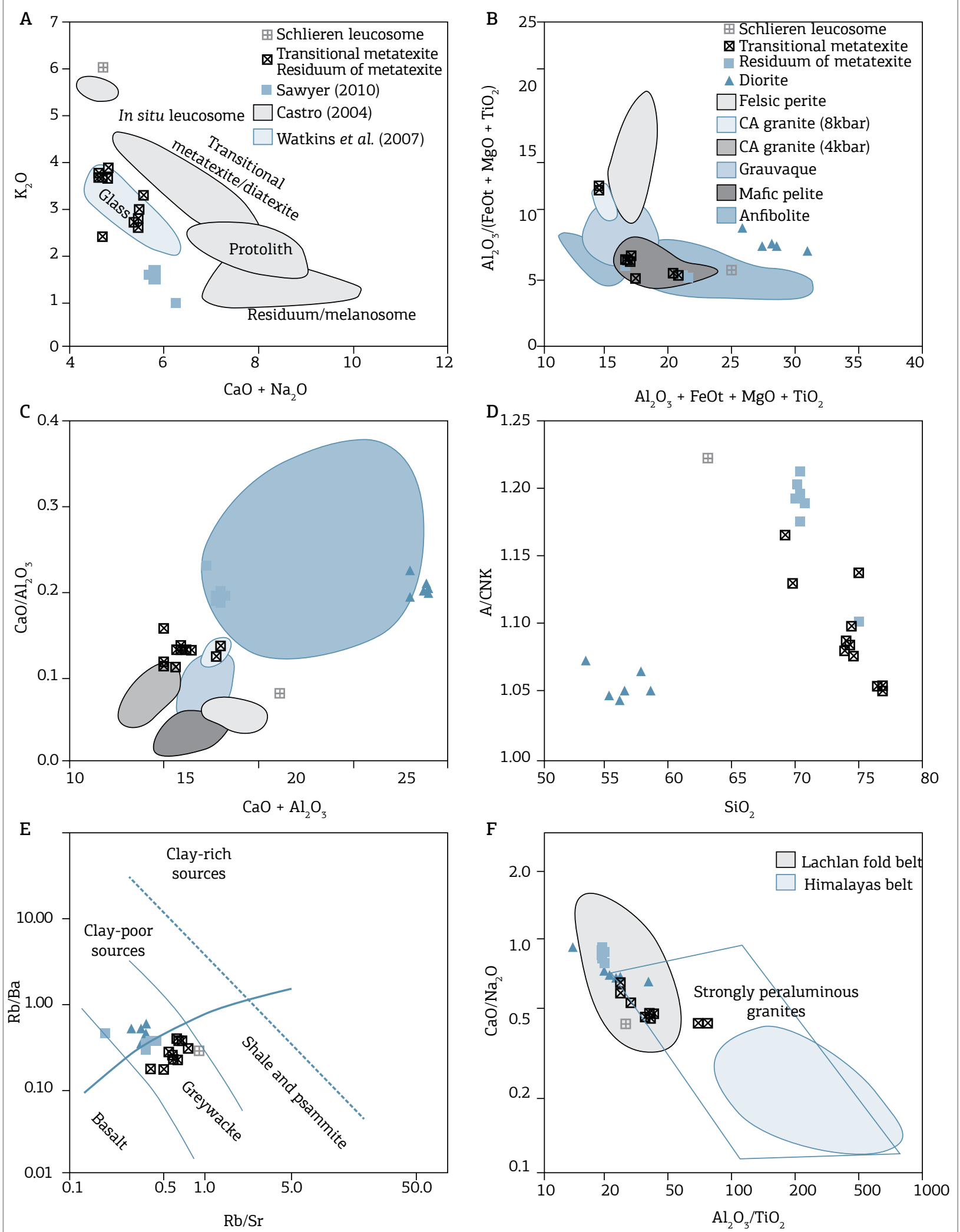

Figure 10. (A) $\mathrm{K}_{2} \mathrm{O}$ versus $\mathrm{CaO}+\mathrm{Na}_{2} \mathrm{O}$ diagram showing compositional similarities between the Furna Azul Migmatite and tonalitic melts; (B and C) Patiño Douce (1999) diagrams; (D) ACNK versus $\mathrm{SiO}_{2}$ diagram; (E and F) Sylvester (1998) diagrams. 


\section{U-PB SHRIMP GEOCHRONOLOGY}

\section{Analytical procedures}

For the U-Pb SHRIMP II analyses, zircon mounts of dioritic injection and stromatic metatexite samples were prepared. In order to select the target spots in the zircon crystals, cathodoluminescence imaging (CL) was obtained by scanning eletron microscopy at the Laboratório de Microscopia Eletrônica de Varredura (Scanning Electron Microscopy Laboratory) of the Centro de Pesquisas Geocronológicas (CPGeo - Geochronological Research Center) of the Universidade de São Paulo. Analytical procedures of U-Pb SHRIMP analysis at CPGeo were described in details by Sato et al. (2014). The reference material was zircon Temora $2(416.8 \pm 3.8 \mathrm{Ma})$ for $\mathrm{U} / \mathrm{Pb}$ ratio calibration and zircon $\mathrm{SL} 13(\mathrm{U}=238 \mathrm{ppm})$ for $\mathrm{U}$ composition. Preferential sites for the analyses were selected so that these were carried out in the CL lighter areas of the zircon and avoiding fractures, when possible. Age calculations were performed with the Isoplot/ EX software of Ludwig (1999). Results are shown with $2 \sigma$ standard deviation.

\section{Results}

\section{Melanosome-rich metatexite (NF54B - 0224766/8267864-UTM)}

Zircons are short prisms (100 to $220 \mu \mathrm{m})$ with smooth apexes, regular magmatic zonation and thin dark borders with common metamictization features (Fig. 12A). The zoned magmatic zircon population yielded $\mathrm{Th} / \mathrm{U}$ ratios from 0.12 to 0.51 and ${ }^{207} \mathrm{~Pb} /{ }^{206} \mathrm{~Pb}$ ages from $1420 \pm 20 \mathrm{Ma}$ to $1479 \pm$ $17 \mathrm{Ma}$ (Tab. 3). A concordant age of $1436 \pm 11 \mathrm{Ma}$ (MSWD $=4.3$ ) was obtained from seven zircon grains (Fig. 12C). One zircon (grain 11.1) furnished a significantly older ${ }^{207} \mathrm{~Pb} /{ }^{206} \mathrm{~Pb}$ age of $1527 \pm 9 \mathrm{Ma}$ and it was not included in the age calculation as it probably represents an inherited zircon or xenocryst.

\section{Dioritic injection \\ (NF42C - 0226563/8267864-UTM)}

The zircon population from dioritic injection shows short to moderately enlongated prisms (200 to $500 \mu \mathrm{m})$ with regular zonation (Fig. 12B). Th/U ratios ranged from 0.05 to 0.43 while ${ }^{207} \mathrm{~Pb} /{ }^{206} \mathrm{~Pb}$ ratios yielded ages from 1337 \pm 6 to $1448 \pm 19 \mathrm{Ma}$ (Tab. 3). In Concordia diagram, the

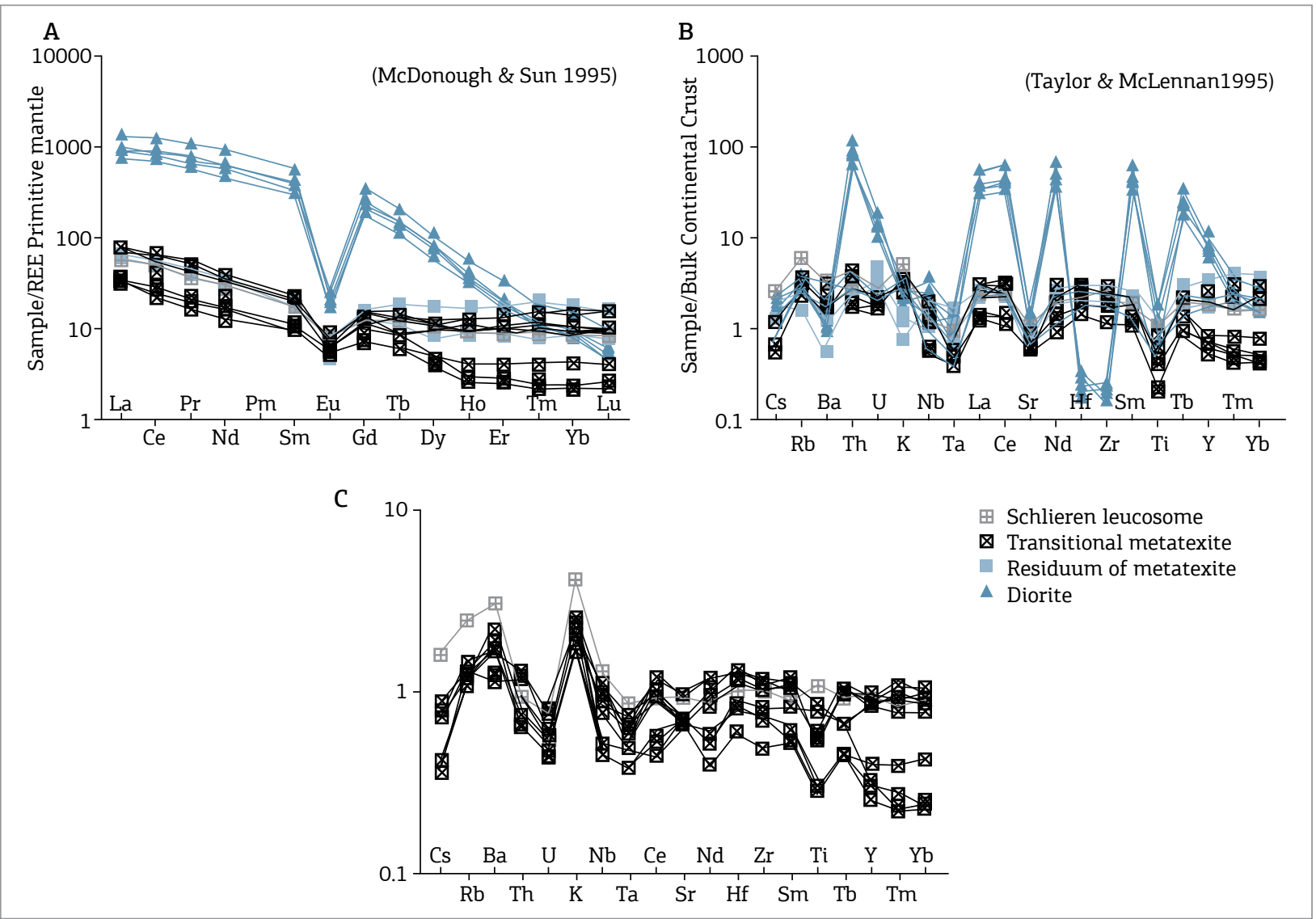

Figure 11. (A) Primitive mantle normalized spidergram; (B) bulk continental crust normalized spidergram; (C) residuum average normalized spidergram. 


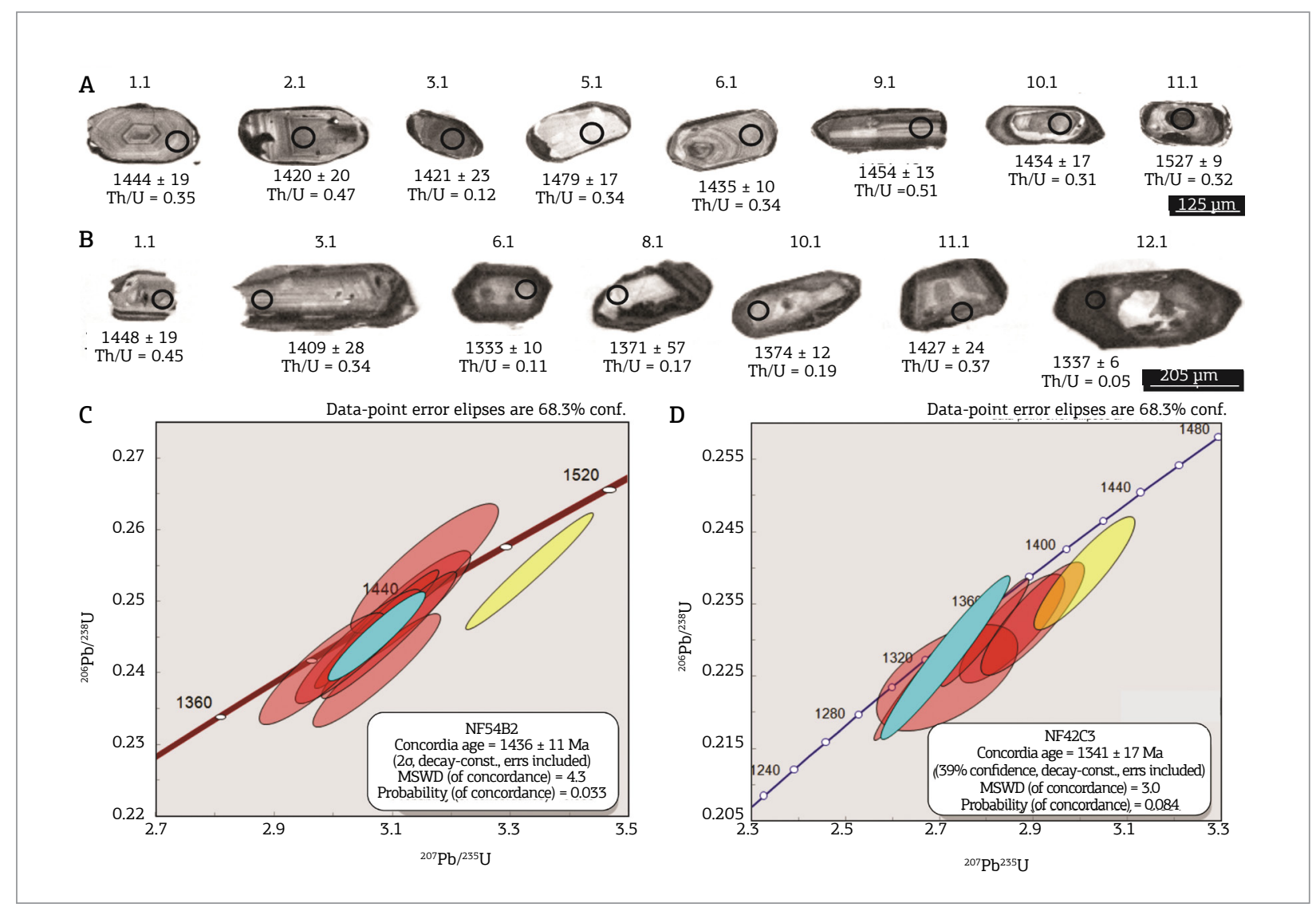

Figure 12. (A) Residuum-rich metatexite (NF-54B) zircon grains; (B) dioritic injection (NF-42C) zircon grains; (C and D) concordia diagram with U-Pb SHRIMP ages for the Furna Azul Migmatite.

Table 3. U-Th-Pb contents, isotopic ratios, agesand their errors (\%) of Furna Azul Migmatite zircon.

\begin{tabular}{|c|c|c|c|c|c|c|c|c|c|c|c|c|c|c|c|c|}
\hline \multirow{2}{*}{$\begin{array}{l}\text { Spot } \\
\text { Name }\end{array}$} & \multirow{2}{*}{$\underset{(\mathrm{ppm})}{\mathrm{U}}$} & \multirow{2}{*}{$\begin{array}{c}\text { Th } \\
\text { (ppm) }\end{array}$} & \multirow[b]{2}{*}{${ }^{232} \mathrm{Th} /{ }^{238} \mathrm{U}$} & \multirow{2}{*}{$\begin{array}{c}f 206 \\
(\%)\end{array}$} & \multicolumn{6}{|c|}{ Radiogenic Ratios } & \multirow[b]{2}{*}{$\begin{array}{c}\text { Err } \\
\text { corr }\end{array}$} & \multicolumn{4}{|c|}{ Age (Ma) } & \multirow{2}{*}{$\begin{array}{c}\% \\
\text { Disc }\end{array}$} \\
\hline & & & & & ${ }^{207} \mathrm{~Pb} /{ }^{206} \mathrm{~Pb}$ & $\begin{array}{c} \pm 1 \sigma \\
(\%)\end{array}$ & ${ }^{207} \mathrm{~Pb} /{ }^{235} \mathrm{U}$ & $\begin{array}{c} \pm 1 \sigma \\
(\%)\end{array}$ & ${ }^{206} \mathrm{~Pb} /{ }^{238} \mathrm{U}$ & $\begin{array}{c} \pm 1 \sigma \\
(\%)\end{array}$ & & ${ }^{206} \mathrm{~Pb} /{ }^{238} \mathrm{U}$ & $\begin{array}{l} \pm 1 \sigma \\
\text { (abs) }\end{array}$ & ${ }^{207} \mathrm{~Pb} /{ }^{206} \mathrm{~Pb}$ & $\begin{array}{l} \pm 1 \sigma \\
\text { (abs) }\end{array}$ & \\
\hline \multicolumn{17}{|c|}{ NF-54B2 - residuum-rich metatexite } \\
\hline 1.1 & 145 & 49 & 0,35 & 0,18 & 0,0909 & 0,98 & 3,1259 & 2,35 & 0,2495 & 2,13 & 0,91 & 1436 & 27 & 1444 & 19 & 0,5 \\
\hline 2.1 & 223 & 101 & 0,47 & 0,18 & 0,0897 & 1,05 & 2,9875 & 2,34 & 0,2415 & 2,09 & 0,89 & 1394 & 26 & 1420 & 20 & 1,8 \\
\hline 3.1 & 393 & 44 & 0,12 & 0,06 & 0,0898 & 1,21 & 3,1593 & 2,58 & 0,2552 & 2,28 & 0,88 & 1465 & 30 & 1421 & 23 & $-3,0$ \\
\hline 5.1 & 133 & 43 & 0,34 & 0,10 & 0,0925 & 0,88 & 3,0787 & 2,30 & 0,2413 & 2,12 & 0,92 & 1393 & 27 & 1479 & 17 & 6,1 \\
\hline 6.1 & 218 & 72 & 0,34 & 0,00 & 0,0905 & 0,55 & 3,0783 & 2,23 & 0,2468 & 2,16 & 0,97 & 1422 & 28 & 1435 & 10 & 0,9 \\
\hline 9.1 & 205 & 100 & 0,51 & 0,10 & 0,0914 & 0,69 & 3,0991 & 2,42 & 0,2460 & 2,32 & 0,96 & 1418 & 30 & 1454 & 13 & 2,6 \\
\hline 10.1 & 244 & 73 & 0,31 & 0,13 & 0,0904 & 0,91 & 3,0480 & 2,28 & 0,2445 & 2,09 & 0,92 & 1410 & 26 & 1434 & 17 & 1,7 \\
\hline 11.1 & 583 & 183 & 0,32 & 0,13 & 0,0949 & 0,47 & 3,3352 & 2,12 & 0,2548 & 2,07 & 0,97 & 1463 & 27 & 1527 & 9 & 4,4 \\
\hline \multicolumn{17}{|c|}{ NF-42C3 - Diorite } \\
\hline 1.1 & 214 & 92 & 0,45 & 0,25 & 0,0911 & 1,01 & 3,0107 & 2,33 & 0,2398 & 2,10 & 0,90 & 1386 & 26 & 1448 & 19 & 4,5 \\
\hline 3.1 & 212 & 70 & 0,34 & 0,66 & 0,0892 & 1,40 & 2,8583 & 2,55 & 0,2323 & 2,09 & 0,82 & 1347 & 25 & 1409 & 28 & 4,7 \\
\hline 6.1 & 437 & 45 & 0,11 & 0,06 & 0,0858 & 0,51 & 2,6523 & 2,14 & 0,2243 & 2,08 & 0,97 & 1304 & 25 & 1333 & 10 & 2,2 \\
\hline 8.1 & 239 & 38 & 0,17 & 1,66 & 0,0875 & 2,80 & 2,7215 & 3,61 & 0,2256 & 2,09 & 0,58 & 1312 & 25 & 1371 & 57 & 4,5 \\
\hline 10.1 & 249 & 46 & 0,19 & 0,09 & 0,0876 & 0,63 & 2,8006 & 2,18 & 0,2318 & 2,08 & 0,96 & 1344 & 25 & 1374 & 12 & 2,3 \\
\hline 11.1 & 232 & 83 & 0,37 & 0,64 & 0,0901 & 1,22 & 2,9015 & 2,50 & 0,2336 & 2,15 & 0,86 & 1354 & 26 & 1427 & 24 & 5,4 \\
\hline 12.1 & 1065 & 55 & 0,05 & 0,04 & 0,0860 & 0,30 & 2,6769 & 2,12 & 0,2258 & 2,10 & 0,99 & 1313 & 25 & 1337 & 6 & 1,9 \\
\hline
\end{tabular}


widespread of ${ }^{207} \mathrm{~Pb} /{ }^{206} \mathrm{~Pb}$ values did not permitted to calculate a concordant age from the seven zircon crystals. Excluding the grain 1.1 , with the older ${ }^{207} \mathrm{~Pb} /{ }^{206} \mathrm{~Pb}$, the other six zircon grains yielded a concordant age of $1341,7 \pm 17 \mathrm{Ma}$ (MSWD $=3.0$; Fig. 12D) which may represent the age of emplacement of the injection. The older ${ }^{207} \mathrm{~Pb} /{ }^{206} \mathrm{~Pb}$ age of $1448 \mathrm{Ma}$ of grain 1.1 can be related to an inherited component in the zircon crystal.

\section{WHOLE ROCK SM-ND ISOTOPE GEOCHEMISTRY}

\section{Analytical procedures}

NF54B (melanosome-rich metatexite) and NF42C (dioritic injection) whole rock samples were pulverized at the Laboratório Multi-Usuário em Técnicas Analíticas (LAMUTA - Analytical Techniques Laboratory) of the Department of Mineral Resources at the Universidade Federal do Mato Grosso, and analyzed at the Laboratório de Geologia Isotópica (Pará-Iso - Isotope Geology Laboratory) of the Universidade Federal do Pará. Sample dissolutions were provided in Savillex ${ }^{\circledR}$ capsules with a mixture of $\mathrm{HNO}_{3}, \mathrm{HCl}$ and $\mathrm{HF}$ after introduction of a ${ }^{149} \mathrm{Sm}-{ }^{150} \mathrm{Nd}$ spike solution. Sm and $\mathrm{Nd}$ extraction was performed by two-step ion exchange chromatography using Biorad Dowex AG $50 \times 8$ resin and Ln Eichron, according to procedures by Gioias and Pimentel (2000) and Oliveira et al. (2008). Isotopic data were acquired with a FINNIGAN MAT262 thermo ionization mass spectrometer. Nd isotopic ratios were corrected of mass discrimination effects using a ${ }^{146} \mathrm{Nd} /{ }^{144} \mathrm{Nd}$ ratio of $0.7219 . \mathrm{Nd} \mathrm{T}_{\mathrm{DM}}$ model ages were calculated using the depleted mantle model of De Paolo (1981).

\section{Results}

$\mathrm{Sm}$ and $\mathrm{Nd}$ isotopic results yielded a $\mathrm{Nd} \mathrm{T}_{\mathrm{DM}}$ model age of $1.90 \mathrm{Ga}$ and a $\varepsilon_{\mathrm{Nd}(1.43 \mathrm{Ga})}$ of -0.54 for the metatexite. Dioritic injection data provided a $\mathrm{Nd} \mathrm{T}_{\mathrm{DM}}$ model age of $1.47 \mathrm{Ga}$ and a $\varepsilon_{\mathrm{Nd}(1.34 \mathrm{Ga})}$ of 3.39 (Table 4).

\section{DISCUSSION AND CONCLUSIONS}

This paper provides the petrographic, geochemical and isotopic characteristics of the Furna Azul Migmatite and associated dioritic injections. New geological and geochronological (U-Pb SHRIMP e Sm-Nd) data are required due to the lack thereof in a key area to the understanding of the Amazon Craton evolution during the Mesoproterozoic Era.

The recognition of migmatites in the region is also important since these rocks can be closely related to orogenic events. Based on petrographic and structural data, transitional metatexites were classified into leucosome-rich and residuum-rich. Leucosome-rich metatexites pointed to higher contents of mobile elements (e.g. K, Rb and $\mathrm{Ba}$ ), whereas residuum-rich metatexites showed higher $U$ contents.

Evidence of metamorphic recrystallization, such as poikiloblastic garnet, sillimanite, biotite symplectic texture and clinopyroxene in mafic enclaves, suggests an upper amphibolite facies condition for the metamorphism. Biotite with prehnite intergrowth has been assumed by Field and Roowell (1968) as originating from metamorphic temperatures between 350 and $450^{\circ} \mathrm{C}$ and low pressure conditions. These conditions are easily achieved through silicate melts and residual rock interactions, under metamorphic conditions. However, the chlorite + muscovite/sericite + carbonate + clay minerals + biotite/prehnite association can be supported by retrograde metamorphism, representing a retrograde trajectory to the greenschist facies.

The lack of garnet + orthopyroxene + cordierite association suggests that partial melting was below the biotite breakdown. These phases are pointed out by Johnson et al. (2008) as peritectic reactions in a graywacke system, as well as in the FAM system.

Metatexites are similar in composition to the anatectic melts of amphibolite, tonalite and graywacke protholiths, as observed in petrographic and geochemical data. They can be geochemically compared to the metagraywackes used by Grapes et al. (2001) to determine the metamorphic grade of quartz-feldspathic rocks. Under low-pressure metamorphism, such rocks can be distinguished between hornblende + (biotite + muscovite + plagioclase \pm garnet) bearing or lacking rocks, while a high metamorphic grade is marked by a lack

Table 4. Sm and Nd whole rock data for the Furna Azul Migmatite samples.

\begin{tabular}{|c|c|c|c|c|c|c|c|c|c|}
\hline Sample & $\begin{array}{c}\text { U-Pb } \\
\text { SHRIMP }\end{array}$ & $\begin{array}{c}\mathrm{Sm} \\
(\mathrm{ppm})\end{array}$ & $\begin{array}{c}\text { Nd } \\
(\mathrm{ppm})\end{array}$ & $\begin{array}{c}{ }^{147} \mathrm{Sm} /{ }^{144} \mathrm{Nd} \\
( \pm 2 \sigma)\end{array}$ & $\begin{array}{c}{ }^{143} \mathrm{Nd} /{ }^{144} \mathrm{Nd} \\
( \pm 2 \sigma)\end{array}$ & $\mathbf{f}_{(\mathrm{Sm} / \mathrm{Nd})}$ & $\varepsilon_{\mathrm{Nd}(0)}$ & $\mathrm{T}_{(\mathrm{DM})}$ & $\varepsilon_{\mathrm{Nd}(\mathrm{t})}$ \\
\hline $\begin{array}{l}\text { NF54B } \\
\text { (metatexite) }\end{array}$ & $1.44 \mathrm{Ga}$ & 9.65 & 46.96 & $0.1243(5)$ & $0.511927(5)$ & -0.368 & -13.8 & 1.90 & -0.54 \\
\hline $\begin{array}{l}\text { NF42C } \\
\text { (diorite) }\end{array}$ & $1.34 \mathrm{Ga}$ & 49.69 & 252.26 & $0.1191(12)$ & $0.512128(4)$ & -0.394 & -9.9 & 1.47 & 3.39 \\
\hline
\end{tabular}


of muscovite and peraluminous granite melt. Therefore, the Patińo Douce and Harris (1998) eaction matches the garnet + sillimanite + melt through biotite breakdown.

The REE pattern of dioritic injections demonstrates both light and heavy REE enrichments, suggesting likely derived from an enriched mantle source, resembling the Sylvester (1998) model of lithospheric delamination and enriched mantle underplating owing to low pressure, and peraluminous and post-collisional magmatism of the Lachlan Fold Belt.

The integration of geological, geochronological and isotopic data points out an age of $1.43 \mathrm{Ga}$ for the metatexite protholith magmatism. The $\mathrm{Sm}-\mathrm{Nd}$ data indicate that this very early igneous event of the San Ignácio Orogeny (1.57$1.30 \mathrm{Ga}$ ) involved the partial melting of an Orosirian continental crust around $1.90 \mathrm{Ga}$.

The following magmatic event, around $1.34 \mathrm{Ga}$, generated dioritic injections through the partial melting of a younger continental crust, extracted from the mantle around 1.47 Ga. According to geochemical and isotopic signatures, a genetic link between the dioritic injections and the metatexite protholith could not be demonstrated.

Regarding the metamorphic and magmatic events of the Paraguá Terrane, the data shown point to a correlation between dioritic injections and the Pensamiento Intrusive Suite (Litherland et al. 1986, Bettencourt et al. 2010, Nalon et al. 2013, França et al. 2014), probably related to syn- to tardi-kinematic granites of a continental arc during the San Ignácio Orogeny.

The age of the Furna Azul Migmatite does not match similar rocks from the Chiquitania Complex and Serra do
Baú Complex, which ranged from 1.78 to $1.72 \mathrm{Ga}$ (Santos et al. 2008, Faria et al. 2014, among others), and with the granulites of Lomas Manechis Complex, with ages between 1.67 and 1.62 Ga (Boger et al. 2005, Matos et al. 2013). Migmatites show protholith age consistent with the San Ramon Granite (1.43 Ga), Bolivia, as presented by Matos Salinas (2010).

The data herein not only analyzed the petrogenetic processes of the Furna Azul Migmatite but also indicated the occurrence of an unknown magmatic event around $1.43 \mathrm{Ga}$ within the Paraguá Terrane, which is worth to be investigated in future studies.

\section{ACKNOWLEDGEMENTS}

The authors thank the National Counsel of Technological and Scientific Development, in Portuguese Conselho Nacional de Desenvolvimento Científico e Tecnológico (CNPq) (Proc. 479779/2011-2), the National Program of Academic Cooperation, or Programa Nacional de Cooperaçáo Acadêmica (PROCAD)/Coordination for the Improvement of Personal Higher Education, or Coordernação de Aperfeiçoamento de Pessoal de Nível Superior (CAPES) (096/2007), the National Institute of Science and Technology (INCT) - Geociam and the Geoscience Postgraduate Program (PPGEC) of the Universidade Federal do Mato Grosso (UFMT). The first author thanks CAPES for granting a Masters scholarship.

\section{REFERENCES}

Bettencourt J.S., Leite W.B.J., Ruiz A.S., Matos R., Payolla B.L., Tosdal R.M. 2010. The Rondonian-San Ignácio Province in the SW Amazonian Craton: an overview. Journal of South American Earth Sciences, 29(1):28-46.

Boger S.D., Raetz M., Giles D., Etchart E., Fanning M.C. 2005. U-Pb age data from the Sunsas region of eastern Bolivia evidence for the allochtonous origin of the Paraguá Block. Precambrian Research, 139:21-146.

Brito Neves B.B. 2011. The Paleoproterozoic in the South-American continent: Diversity in the geologic time. Journal of South American Earth Sciences, 32:270-286.

Castro A. 2004. The source of granites: inferences from the Lewisian complex. Scottish Journal of Geology, 40:4-65.

Cordani U.G. \& Teixeira W. 2007. Proterozoic accretionary belts in the Amazonian Craton. 4-D Framework of Continental Crust, 200:297-320.

Cordani U.G., Tassinari C.G.C., Teixeira W., Basei M.A.S., Kawashita K. 1979. Evolução tectônica da Amazônia com base nos dados geocronológicos. In: Congreso Geológico Chileno, 2, Arica. Short Paper, p. 137-138.
Cordani U.G., Teixeira W., D’Agrella-Filho M.S., Trindade R.I. 2009. The position of the Amazonian Craton in supercontinents. Gondwana Research, 15:396-407.

Cox K.G., Bell J.D., Pankhurst R.J. 1979. The interpretation of igneous rocks. London, George Allen \& Unwin, 450 p.

De Paolo D.J. 1981. Nd isotopic studies: some new perspectives on Earth structure and evolution. EOS, 62(14):137-145.

Faria D.A., Ruiz A.S., Matos J.B., Sousa M.Z.A., Lima G.A., Moacir J.B.M. 2014. Geology geochemistry and geochronology (U-Pb) of the Rio Fortuna Gneiss - Serra do Baú Intrusive Suite - Paraguá Terrane SW Amazonian Craton. Brazilian Journal of Geology, 44(1):139-154.

Fettes D. \& Desmons. J. (Eds.). 2008. Metamorphic rocks: a classification and glossary of terms. Cambridge, Cambridge University Press, 243 p.

Field D. \& Roowell J.R. 1968. The occurrence of prehnite in a high grade metamorphic sequence from South Norway. Norsk Geografisk Tidsskrift - Norwegian Journal of Geograph, Oslo, 48:55-59.

Figueiredo F.L.P., Ruiz A.S., Sousa M.Z.A., Macambira M.J.B. 2013. Ortognaisse Turvo: registro de magmatismo Paleoproterozóico no Terreno Paraguá - SW do Cráton Amazônico. Brazilian Journal of Geology, 43(2):401-422. 
França O., Ruiz A.S., Sousa M.Z.A., Batata M.E.F., Lafon J.-M. 2014. Geology, petrology U-Pb (shrimp) and geochronology of the Morrinhos granite - Paraguá terrane. SW Amazonian craton: implications for the magmatic evolution of the San Ignácio orogeny. Brazilian Journal of Geology, 44(3):415-432.

Frost B.R., Barnes C.G., Collins W.J., Arculus R.J., Ellis D.J., Frost C.D. 2001. A geochemical classification for granitic rocks. Jounal of Petrology, 42:2033-2048

Fuck R.A., Brito Neves B.B., Schobbenhaus C. 2008. Rodinia descendants in South America. Precambrian Research, 160:108-126.

Geraldes M.C., Van Schmus W.R., Condie K.C., Bell S., Teixeira W., Babinski M. 2001. Proterozoic geologic evolution of the SW part of the Amazonian Cráton in Mato Grosso state. Brazil. Precambrian Research, 111:91-128.

Gioias M.C.L. \& Pimentel M.M. 2000. The Sm-Nd isotopic method in the Geochronology Laboratory of the University of Brasília. Anais da Academia Brasileira de Ciências, 72(2):219-245

Grapes R., Roser B., Kifle K. 2001. Composition of monocrystalline detrital and authigenic minerals metamorphic grade and provenance of Torlesse and Waipapa greywacke. Central North island. New Zealand. International Geology Review, 43:139-175.

Harker A. 1909. The natural history of the igneous rocks. New York, The Macmillan Company, $384 \mathrm{p}$.

Harris N.B.W., Pearce J.A., Tindle A.G. 1986. Geochemical characteristics of collision-zone magmatism. Special Publications of Geological Society, London, 19:67-81.

Holmquist P.J. 1916. Swedish Archeaen structures and their meaning. Bulletin of the Geological Institute Upsala, 15:125-148.

Irvine I.N. \& Baragar W.R.A. 1971. A guide to the chemical classification of the common volcanic rocks. Canadian Journal Earth Science, 8:523-548.

Janoušek V., Farrow C.M., Erban V., Trubač J. 2011. Brand new Geochemical Data Toolkit (GCDkit 3.0) - is it worth upgrading and browsing documentation? (Yes!). Geologickevyzkumy na Morave a veSlezsku, 18:26-30.

Jesus G.C., Sousa M.Z.A., Ruiz A.S., Matos J.B. 2010. Petrologia e geocronologia (U/Pb-Sm/Nd) do Granito Passagem. Complexo Granitóide Pensamiento. SW do Cráton Amazônico (MT). Revista Brasileira de Geociências, 40(3):392-408.

Johnson T.E., White R.W., Powell R. 2008. Partial melting of metagreywacke: a calculated mineral equilibria study. Journal Metamorphic Geology, 26:837-853.

Litherland M. \& Bloomfield K. 1981. The Proterozoic history of eastern Bolivia. Precambrian Research, 15:157-179.

Litherland M., Annels R.N., Darbishire D.P.F., Fletcher C.J.N., Appleton J.D., Berrangé J.P., Bloofield K., Burton C.C.J., Hawkin M.P., Klinck B.A., Llanos A., Mitchell W.I., O'Connor E.A., Pitfield P.E.J., Power G., Webb B.C. 1986. The geology and mineral resources of the Bolivian Precambrian Shield. London, British Geological Survey, Natural Enviroment Research Council, 153 p.

Ludwig K. R. 1999. Isoplot/Ex 2.01: a geochronological toolkit for Microsoft Excel. Berkeley Geochronology Center Special Publication, $50 \mathrm{p}$.

Matos J.B., Juliani C., Tokashiki C.C., Oliveira R.F., Ruiz A.S. 2013. Granulitos ortoderivados da Suíte Lomas Manechis. Fronteira BrasilBolivia - Geoquimica e Geocronologia. In: Simpósio de Geologia da Amazônia, 13, Belém. Short Paper, p. 305-308.

Matos J.B., Ruiz A.S., Sousa M.Z.A., Batata M.E.F., Lima G.A. Em preparação. Petrologia. Geoquímica e Geocronologia U/Pb e Sm/Nd do Granito Tarumã. Terreno Paraguá - Fronteira Brasil-Bolívia. In: Contribuições à Geologia da Amazônia, v. 10.
Matos R., Teixeira W., Geraldes M.C. 2008. El granito diamantina: evidencia isotópica y química de magmatismo de arco em El Complejo Pensamiento. Província Rondoniana-San Ignacio. Precâmbrico de Bolivia oriental. Revista del Instituto de Investigaciones Geológicas y del Medio Ambiente, 2:5-11.

Matos R., Teixeira W., Geraldes M.C., Bettencourt J.S. 2009. Geochemistry and Nd-Sr isotopic signatures of the Pensamiento Granitoid Complex. Rondonian-San Ignacio Province. Eastern Precambriam shield of Bolivia: petrogenetic constraints for a Mesoproterozoic Magmatic Arc Setting. Geologia USP, Série Científica, 9(2):89-117.

Matos Salinas G.R. 2010. Geocronologia e evolução tectônica paleo-mesoproterozoica do oriente boliviano - região sudoeste do cráton amazônico. PhD Thesis, Universidade de São Paulo, São Paulo, $52 \mathrm{p}$.

McDonough W.F. \& Sun S.S. 1995. Composition of the Earth. Chemical Geology, 120:223-253.

Mehnert K.R. 1968. Migmatites and the origin of granitic rocks. Amsterdam, London, New York, Price, Elsevier, 393 p.

Nalon P.A., Sousa M.Z.A., Ruiz A.S., Macambira M.J.B. 2013. Batólito Guaporé́: uma extensão do Complexo Granitóide Pensamiento em Mato Grosso - SW do Cráton Amazônico. Brazilian Journal of Geology, 43(1):85-100.

Nascimento N.D.C., Campos F.A.P., Ruiz A.S., Souza M.Z.A., Pierosan R., Alonso R.V. 2013. Petrografia e deformação do Gnaisse Furna Azul. Suíte Intrusiva Serra do Baú - Terreno Paraguá - SW do Cráton Amazônico. In: Simpósio Nacional de Estudos Tectônicos, 14, Chapada dos Guimarães. Anais..., CD-ROM.

Oliveira E.C., Lafon J.M., Gioia S.M.L., Pimentel M.M. 2008. Datação Sm-Nd em rocha total e granada do metamorfismo granulítico da região de Tartarugal Grande. Amapá Central. Revista Brasileira de Geociências, 38:116-129.

Patiño Douce A.E. 1999. What do experiments tell us about the relative contributions of crust and mantle to the origin of granitic magmas? Geological Society, London, Special Publications, 168:55-75.

Patiño Douce A.E. \& Harris N. 1998. Experimental constraints on Himalayan Anatexis. Journal of Petrology, 39:689-710.

Ruiz A.S. 2005. Evolução geológica do sudoeste do Cráton Amazônico região limitrofe Brasil-Bolívia-Mato Grosso. PhD Thesis. Universidade Estadual Paulista "Júlio de Mesquita Filho", Rio Claro, 260 p.

Ruiz A.S., Matos J.B., Sousa M.Z.A., Lima G.A., Macambira M.B., Matos G.R., Faria D.A., Fança O., Costa P.C.C. 2012. Granites of Pensamiento Intrusive Suite: records of Continental Magmatic Arc San Ignacio in Brazil. In: South American Symposium on Isotope Geology, 8, Medellín. Short Paper.

Ruiz A.S., Sousa M.Z.A., Matos J.B., Macambira M.B., Lima G.A., Faria D.A. 2011. Cráton ou Terreno Paraguá? Uma discussão baseada em novos dados geológicos e geocronológicos do SW do Cráton Amazônico em território brasileiro. In: Simpósio Nacional de Estudos Tectônicos, 13, Campinas. Short Paper, p. 239-242.

Santos J.O.S., Rizzotto G.J., Potter P.E., McNaughton N.J., Matos R.S., Hartmann L.A., Chemale F., Quadros M.E.S. 2008. Age and autochthonous evolution of the Sunsás Orogen in West Amazon Craton based on mapping and $\mathrm{U}-\mathrm{Pb}$ geochronology. Precambriam Research, 165:120-152.

Sato K., Tassinari C.C.G., Basei M.A.S., Siga Jr. O., Onoe A.T., Souza M.D. 2014. Sensitive High Resolution Ion Microprobe (SHRIMP IIe/MC) of the Institute of Geosciences of the University of São Paulo. Brazil: analytical method and first results. Geologia USP, Série Cientifica, 14(3):3-18. 
Sawyer E.W. 2008.Working with migmatites: nomenclature for the constituent parts. In: Sawyer E.W. \& Brown M. (Eds.). Working with migmatite: Mineralogical Association of Canada. Short Course, 38:1-28.

Sawyer E.W. 2010. Migmatites formed by water-fluxed partial melting of a leucogranodiorite protolith: microstructures in the residual rocks and source of the fluid. Lithos, 116:273-286

Sederholm J.J. 1907. Om granitochgneis. Bulletin de la Commission Géologique de Finlande, 23:1-110.

Shand S.J. 1943. Eruptive rocks their genesis, composition, classification and their relation to ore deposits with a chapter on meteorite. New York, John Wiley \& Sons.

Sylvester P.J. 1998. Post-collisional strongly peraluminous granites. Lithos, 45:29-44.

Tassinari C.C.G. \& Macambira M.J.B. 1999. Geochronological provinces of the Amazonian Craton. Episodes, 22:174-182.
Tassinari C.C.G., Bettencourt J.S., Geraldes M.C., Macambira M.J.B., Lafon J.M. 2000. The Amazonian Craton. In: Cordani U.G., Milani E.J. Filho A.T., Campos D.A. (Eds.) Tectonic evolution of South America. Rio de Janeiro, 31 $1^{\text {st }}$ International Geological Congress, p. 41-95.

Taylor S.R. \& McLennan S.M. 1995. The geochemical evolution of the continental crust. Reviews in Geophysics, 33:241-265.

Teixeira W., Geraldes M.C., Matos R., Ruiz A.S., Saes G., Vargas-Matos G. 2010. A review of the tectonic evolution of the Sunsás belt - SW portion of the Amazonian Craton. Journal of South American Earth Science, 29:47-60.

Teixeira W., Tassinari C.C.G., Cordani U.G., Kawashita K. 1989. A review of the geochronology of the Amazonian Craton: tectonic implications. Precambrian Research, 42:213-227.

Watkins J.M., Clemens J.D., Treloar P.J. 2007. Archaean TTGs as sources of younger granitic magmas: melting of sodic metatonalites at 0.61.2 GPa. Contributions to Mineralogy and Petrology, 154:91-110.

Available at www.sbgeo.org.br 